\title{
Cavity formation on the surface of a body entering water with deceleration
}

\author{
M. Reinhard • A. A. Korobkin • \\ M. J. Cooker
}

Received: date / Accepted: date

\begin{abstract}
The two-dimensional water entry of a rigid symmetric body with account for cavity formation on the body surface is studied. Initially the liquid is at rest and occupies the lower half-plane. The rigid symmetric body touches the liquid free surface at a single point and then starts suddenly to penetrate the liquid vertically with a time-varying speed. We study the effect of the body deceleration on the pressure distribution in the flow region. It is shown that, in addition to the high pressures expected from the theory of impact, the pressure on the body surface can later decrease to sub-atmospheric levels. The creation of a cavity due to such low pressures is considered. The cavity starts at the lowest point of the body and spreads along the body surface forming a thin space between a new free surface and the body. Within the linearised hydrodynamic problem, the positions of the two turnover points at the periphery of the wetted area are determined by Wagner's condition. The ends of the cavity's free surface are modelled by the Brillouin-Villat condition. The pressure in the cavity is assumed to be a prescribed constant, which is a parameter of the model. The hydrodynamic problem is reduced to a system of integral and differential equations with respect to several functions of time. Results are presented for constant deceleration of two body shapes: a parabola and a wedge. The general formulation made also embraces conditions where the body is free to decelerate under the total fluid force. Contrasts are drawn between results from the present model and a simpler model in which the cavity formation is suppressed. It is shown that the expansion of the cavity can be significantly slower than the expansion of the corresponding zone of sub-atmospheric pressure in the simpler model. For forced motion and cavity
\end{abstract}

M. Reinhard

IRTG 1529, Universität Darmstadt, Germany

E-mail: moritz.reinhard@gmail.com, reinhard@mathematik.tu-darmstadt.de

A. A. Korobkin, M. J. Cooker

School of Mathematics, University of East Anglia, Norwich, NR4 7TJ, England

E-mail: a.korobkin@uea.ac.uk,m.cooker@uea.ac.uk 
pressure close to atmospheric, the cavity grows until almost complete detachment of the fluid from the body. In the problem of free motion of the body, cavitation with vapour pressure in the cavity is achievable only for extremely large impact velocities.

Keywords Solid-liquid impact · water entry · Wagner model · cavitation

\section{Introduction}

For a rigid body impacting a water free surface, the body experiences large hydrodynamic forces especially if the deadrise angle between the structure and the initial flat water surface is small. However, when the body velocity decreases during impact, high pressures occur only close to the periphery of the contact region, and pressures below atmospheric may occur well inside of the contact region. For example, the free fall of a blunt body has been analysed in $\S 2.3 .3$ of [16]. Due to the large hydrodynamic loads a light blunt body significantly decelerates and equations in [16] show that the hydrodynamic pressures become sub-atmospheric. For quickly decelerating bodies the pressure in the contact region may be so low that the fluid cavitates at the fluid-body interface (see Figure 1). In theory, cavities develop as soon as the pressure drops below the vapour pressure of water: for water at $20^{\circ}$ Celsius, Kaye and Laby [7] report the vapour pressure is $2.3 \mathrm{kPa}$, which is very small compared with $101 \mathrm{kPa}$ for atmospheric pressure. In practice, cavities may develop at fluid pressures higher than the vapour pressure when there are air bubbles already present in the fluid able to grow in such low pressure zones. Cavitation is an important issue in ship hydrodynamics and was reported in drop tests of horizontal elastic plates on a flat water surface [2].

The phenomenon of high pressure pockets of trapped air forming in bodyfluid impact problems, also called air-cushioning, is related to cavitation. This phenomenon is due to the squeezed air in the small gap between fluid and body shortly before impact. A large volume of air is trapped if the shape of the bottom of the body is of low curvature. In that case, the trapped air influences the hydrodynamic loads on the body. Important contributions in the modelling of air-cushioning are given by [4], [6], [20] and [22]. Note that for an air-cushioning model the dynamics of the air film between body and fluid is crucial. In order to find the shape of the air-pocket, the air-water interaction needs to be investigated in the stage when the body approaches the fluid surface. In contrast, a low-pressure cavity starts to form when the body surface is already in contact with the fluid.

Experimental results with a light circular disc impacting the water surface were reported by [13]. They showed that a cavity is formed on the lower surface of the disc. A thin rod with a sharp end was put in the water vertically at the centre line of the disc. The sharp end of the rod was set well below the water surface. The disc was decelerated by the hydrodynamic force before it came into contact with the rod. Next, the disc was halted by the rod instantaneously, after which the cavity's free surface separated from the disc, and grew as an 
expanding hemisphere. These experiments were designed to demonstrate that the inertia of the fluid is able to separate the fluid from the surface of the body that is penetrating the fluid with deceleration.

We are concerned with impact problems, where the contact region of water and body is of the order of $1 \mathrm{~m}$ and the vertical speed of the body is of order $1 \mathrm{~ms}^{-1}$. Due to the low kinematic viscosity of water, the Reynolds number is of order $10^{6}$. We are aware of the roles of viscosity even for such large Reynolds numbers in the fluid detachment from the body in the low-pressure zone. It should be noted that in some problems of water entry the viscosity may play the dominant role. This is the case for the almost vertical entry of a thin body, as stressed in [12]. Viscous effects are not included in the present model. However, its role is needed be identified in future research.

Our aim is to analyse the development of a cavity during vertical impact of bodies of general symmetric shapes within the Wagner theory of fluid impact due to [21]. Note that the Wagner theory considers only body surfaces with small deadrise angles. In order to take cavities into account, one needs boundary conditions on the fluid-body interface. The account of a cavity in symmetric-body impact has been studied in [10] where atmospheric pressure was imposed in the cavity region. In the present paper, we analyse a generalised problem of Korobkin [10] by assuming the cavitation pressure to be constant and not necessarily equal to atmospheric pressure. The flow is complicated since the position of the free surface in the cavity region and the contact points are unknown. Korobkin [10] only presented asymptotic results for the initial stage of cavity formation, and this motivated us to calculate the evolution of the cavity numerically. Here we reduce the flow problem to an initial-value problem with respect to unknown coefficients that evolve in time. We confirm the asymptotic results of [10] for the initial stage of cavity formation, and give numerical results for times when the length of the cavity region becomes comparable with the size of the contact region. We will compare the model where a cavity is accounted for with a simpler model in which cavity formation is suppressed. It is shown that the expansion of a cavity can be significantly slower than the expansion of the corresponding subatmospheric zone in the simpler model. Furthermore, the hydrodynamic upward force exerted is greater with a cavity than without a cavity. In addition to impact problems with prescribed motion we analyse problems where the body freely enters the water by modelling the motion with Newton's second law.

The method presented here can be extended to problems of oblique body impact. Moore et al. [14], [15] and Reinhard et al [17] showed for two and three-dimensional blunt bodies that pressures far below atmospheric appear on the rear part of the wetted region which extends to the rear turnover region. It is also related to patch cavitation [5]. Patch cavitation occurs when a thin cavity lies close to the shoulder of a blunt body immersed in a streaming flow of liquid.

The structure of the paper is as follows. We start with the mathematical formulation of the problem in $\S 2$. The two-dimensional flow generated by the body impact is governed by a mixed boundary-value problem for the veloc- 


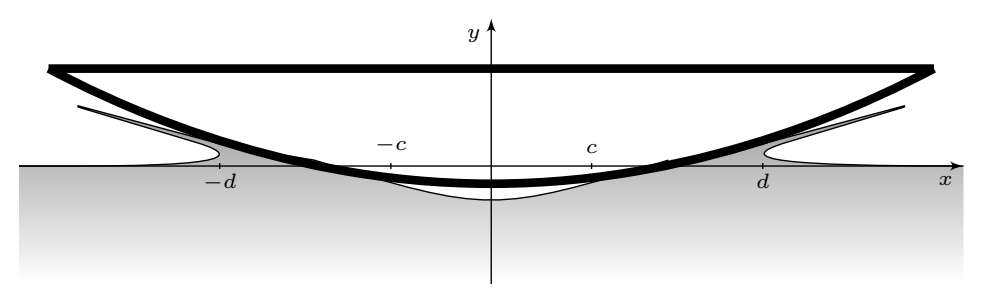

Fig. 1 Impact of a symmetric body onto deep water at time $t>t_{0}$ when a cavity develops at the bottom of the body. The spatial position of the cavity is $|x|<c$. Note that the fluid free surface overturns at $x= \pm d$ from where jets are emanated.

ity potential. We impose Wagner's condition at the turnover regions, Kutta's condition and the condition of continuous free-surface separation at the inner contact points. We also ensure that the fluid cannot penetrate the body and that the hydrodynamic pressure is not below the cavity pressure. The solution of the problem is outlined in $\S 3$ for the initial entry stage without a cavity and in $\S 4$ for the subsequent stage when the cavity develops. Six equations for six unknown functions are derived in $\S 4$ by comparing the acceleration potential, velocity potential and displacement potential at the outer and inner contact points and in the far field. In $\S 5$ numerical results are shown for the impact of a parabola and wedge under constant deceleration. $\S 6$ explains how to apply the present theory to the problem when a body falls freely on the water surface and the body responds to the hydrodynamic force. Conclusions are drawn and future work is suggested in $\S 7$.

\section{Mathematical formulation}

Initially the incompressible and inviscid liquid is at rest and occupies the lower half plane $y<0$ of a fixed Cartesian coordinate system $x O y$. The body initially touches the free surface at a single point which is taken as the origin of the coordinate system. At $t=0$ the body starts to penetrate the liquid vertically with initial speed $V$. The flow generated by the penetrating body is assumed two-dimensional and irrotational. Gravity and surface tension are neglected in the hydrodynamic model. The inertia of the air is neglected but the influence of its pressure is included in that the atmospheric pressure adjacent to the liquid free surface is set to zero. The position of the body surface is

$$
y=f(x)-h(t)
$$

where the function $f(x)$ describes the shape of the body and $h(t)$ is the penetration depth. Initially, the penetration depth is zero, $h(0)=0$. The penetration depth $h(t)$ is first a prescribed function but later it will be the solution of an equation of motion. The shape function $f(x)$ is even, $f(-x)=f(x)$, and its first derivative $f^{\prime}(x)$ is small in the vicinity of the bottom point $f(0)=0$. It follows from the symmetric shape of the body and the symmetric geometry of 
the initial hydrodynamic configuration, that the generated flow is symmetric with respect to the $y$-axis. We are concerned with the initial stage of body impact where the horizontal extension of the wetted region of the body is much larger than the penetration depth.

Figure 1 shows the symmetric body as it penetrates the fluid free surface with a cavity below the body. The initiation of a cavity in water is physically complex and depends on the tensile strength of water, and hence on the degree of aeration, dissolved gases and solid nuclei in the water. Here we take as simple as possible a view of the continuum model. We assume that there is no cavity until such time as the pressure distribution on the body possesses a spatial minimum that falls below a pre-set value $p_{c}$. Here $p_{c}$ is referred to as the cavitation pressure. We know that $p_{c}$ depends on the vapour mass content and the area of the cavity. But for simplicity we only show results where $p_{c}$ is fixed to be a non-positive constant. We assume that the cavity grows from the lowest point $x=0$ on the body and that the cavity is a simply-connected region whose free surface is the graph of a single-valued function of $x$. Note that two spray jets are shown in Figure 1. These jets are not included in the model of this paper. These jets were studied in [6] where the conditions of the jets separation from the surface of the body were obtained.

When the penetration depth is small compared to the dimension of the wetted part of the entering contour, the boundary conditions can be linearised and projected onto the position of the undisturbed free surface, $y=0$. A formal linearisation and a discussion of the asymptotic structure can be found in [16]. The set of points $x \in R_{f}, y=0$, corresponds to the free surface outside the wetted region, and $x \in R_{w}, y=0$, corresponds to the wetted part of the impacting body. The position of the cavity region is described by $x \in R_{c}, y=0$. Like in [10] we distinguish two stages. During the first stage the hydrodynamic pressure on the body is above the cavitation pressure. In this stage we have no cavity so that the body is wetted along a single interval and we set

$$
R_{f}=\{x:|x|>d\}, \quad R_{w}=\{x:|x|<d\}, \quad R_{c}=\emptyset .
$$

This stage is referred to as the Wagner stage. The points $x=-d(t), y=0$ and $x=d(t), y=0$ are the overturning points referred to below as the Wagner contact points. We only consider impact problems where the entry velocity $\dot{h}(t)$, where dot stands for the time derivative, is positive during the Wagner stage.

The second stage starts at the time instant $t=t_{0}$ when the pressure at the centre of the body surface has first decreased to cavitation pressure $p_{c}$. In this stage of impact a cavity grows on the body-fluid interface from $x=0$ and splits the wetted part of the body into two intervals. This stage is referred to as the cavitation stage and we set

$$
R_{f}=\{x:|x|>d\}, \quad R_{w}=\{x: c<|x|<d\}, \quad R_{c}=\{x:|x|<c\} .
$$

Here $x=-c(t), y=0$ and $x=c(t), y=0$ correspond to the points where the cavity surface meets the body. These points are referred to below as the 
separation points. The functions $c(t)$ and $d(t)$ are unknown in advance and are determined as part of the solution. The speeds $\dot{c}$ and $\dot{d}$ are assumed positive during the early stage.

The irrotational velocity field is described by the velocity potential $\phi(x, y, t)$. The hydrodynamic pressure is given by Bernoulli's equation, which is linearised during the initial stage of impact:

$$
p(x, y, t)=-\varrho \phi_{t}(x, y, t) \quad(y \leq 0),
$$

where $\varrho$ is the constant fluid density. Within this model, which is referred to as the Wagner model of water impact [21], the thin jets thrown outwards from $x= \pm d(t)$, sketched in Figure 1, are not taken into consideration. The flow in the jet region can be found at leading order after the flow in the main region has been obtained [6]. The velocity potential $\phi(x, y, t)$ satisfies the following mixed boundary-value problem:

$$
\begin{aligned}
\nabla^{2} \phi & =0 \quad(y<0), \\
\phi_{y} & =-\dot{h} \quad\left(y=0, x \in R_{w}\right), \\
\phi_{t} & =0 \quad\left(y=0, x \in R_{f}\right), \\
\phi_{t} & =-p_{c} / \varrho \quad\left(y=0, x \in R_{c}\right), \\
\phi & =O\left(r^{-1}\right) \quad\left(r=\sqrt{x^{2}+y^{2}} \rightarrow \infty\right) .
\end{aligned}
$$

Equations (7) and (8) follow from Bernoulli's equation (4) together with the dynamic boundary conditions: $p=0$ on the outer free surface and $p=p_{c}$ on the free surface in the cavity region. Equation (6) is the linearised body boundary condition. The linearised problem (5) - (9) breaks down close to the Wagner contact points. Inner solutions in these regions given by the Kelvin-Helmholtz cavity flow provide the following singular behaviour of the flow velocity in the outer problem (see [6]):

$$
|\nabla \phi|=O\left(r_{ \pm d}^{-1 / 2}\right) \quad\left(r_{ \pm d}=\sqrt{(x \mp d)^{2}+y^{2}} \rightarrow 0\right) .
$$

We seek a velocity potential $\phi(x, y, t)$ which satisfies $(5)-(10)$. The function $d(t)$ is determined by using Wagner's condition that the splash is so thin that the free surface meets the body at the turnover point [21]:

$$
\eta(x, t)=f(x)-h(t) \quad(x= \pm d),
$$

where $y=\eta(x, t)$ is the free-surface elevation for $x \in R_{f} \cup R_{c}$. The elevation $\eta(x, t)$ is obtained from the time-integral of the vertical velocity component in the linearised free-surface kinematic boundary conditions

$$
\eta_{t}(x, t)=\phi_{y}(x, 0, t) \quad\left(x \in R_{f} \cup R_{c}\right)
$$

by using the initial condition $\eta(x, 0) \equiv 0$ and assuming continuity of the fluid surface at $x= \pm c$ :

$$
\eta(x, t)=f(x)-h(t) \quad(x= \pm c) .
$$


Note the similarity of the approximate condition (11) and the exact condition (13).

During the cavitation stage the function $c(t)$ is determined with the help of Kutta's condition at $x= \pm c$

$$
|\nabla \phi( \pm c, 0, t)|<+\infty
$$

and the two inequalities

$$
\begin{aligned}
\eta(x, t) & \leq f(x)-h(t) \quad\left(x \in R_{c}\right), \\
p(x, 0, t) & \geq p_{c} \quad\left(x \in R_{w}\right) .
\end{aligned}
$$

Kutta's condition models a smooth flow separation from the body and a continuous pressure distribution at the contact points $x= \pm c, y=0$. Inequalities (15) and (16) imply that the liquid particles cannot penetrate the rigid surface and that the hydrodynamic pressure in the contact region cannot be less than the cavitation pressure. Both inequalities were used in [5], [8] and [18].

The solution of the problem (4)-(16) depends on the fluid density $\varrho$, the cavitation pressure $p_{c}$, the body shape described by the function $f(x)$ and the vertical motion of the body, $h(t)$. The analysis will be focused on the motion of the contact points, the hydrodynamic loads and the shape of the cavity region. A direct numerical solution is difficult because the problem is nonlinear, coupled and unknown functions are singular. We start with the Wagner stage $0<t<t_{0}$ where we consider equations (4)-(12) for the flow regime without a cavity. Results at the end of the Wagner stage are used as initial conditions for the problem in the cavitation stage.

\section{Wagner stage}

We consider the solution of the problem (4)-(12) in the Wagner stage $0<t<$ $t_{0}$. The Wagner condition (11) has been transformed by [9] and reduced to the equation

$$
\int_{0}^{\pi / 2} f(d(t) \sin \theta) \mathrm{d} \theta=\frac{\pi}{2} h(t),
$$

which determines the positions of the Wagner contact points $x= \pm d(t)$. Furthermore, equations (4) - (9) give us the hydrodynamic pressure on the wetted part of the body [16]:

$$
p(x, 0, t)=\frac{\varrho}{\sqrt{d^{2}-x^{2}}}\left(\ddot{h}\left(d^{2}-x^{2}\right)+\dot{h} \dot{d} d\right) \quad(|x|<d(t)) .
$$

The pressure (18) in the contact region takes its minimum value at the centre $x=0$, so that the cavitation stage starts at $t=t_{0}$ when the following equation is satisfied:

$$
p_{c}=\varrho(\ddot{h} d+\dot{h} \dot{d})
$$


Since the entry velocity is considered to be positive in the Wagner stage and $\dot{d}>0$ equation (19) implies that a cavity only can develop while the body decelerates, $\ddot{h}<0$.

As mentioned in the previous section, the Wagner theory can be used only for small penetration depths. One needs to check that $h / d \ll 1$ is still satisfied at $t=t_{0}$. Only then is the analysis in the next section valid.

\section{Cavitation stage}

In this section we introduce the acceleration and displacement potential and reformulate the hydrodynamic problem $(5)$ - (9) with respect to these potentials. Apart from the positions of the contact points, $x= \pm c$ and $x= \pm d$, the solutions of all three mixed-boundary value problems in the lower half plane involve four further unknowns which come from eigensolutions of the problems and time-integration. Equations for the six unknowns are obtained by comparing the behaviour of all three potentials at the inner contact point $x=c$, $y=0$, at the outer contact point $x=d, y=0$ and in the far-field $x^{2}+y^{2} \rightarrow \infty$. Furthermore, we will discuss in the present section how to calculate the hydrodynamic pressure and force. Finally, we investigate the asymptotic flow behaviour at the initial stage of cavity formation.

\subsection{Acceleration potential}

The acceleration potential is defined by $\varphi=\phi_{t}$ and we obtain the hydrodynamic problem with respect to this potential from the mixed boundary value problem (5) - (9). Hence,

$$
\begin{aligned}
\nabla^{2} \varphi & =0 \quad(y<0) \\
\varphi_{y} & =-\ddot{h} \quad(y=0, c<|x|<d) \\
\varphi & =0 \quad(y=0,|x|>d) \\
\varphi & =-p_{c} / \varrho \quad(y=0,|x|<c) \\
\varphi & =O\left(r^{-1}\right) \quad\left(r=\sqrt{x^{2}+y^{2}} \rightarrow \infty\right) .
\end{aligned}
$$

Since we know that Kutta's condition (14) is satisfied at the inner contact points and, that the velocity potential satisfies condition (10), we obtain the asymptotic behaviours of $\nabla \varphi$ at $x= \pm c, \pm d, y=0$ (see [19]):

$$
\begin{array}{ll}
|\nabla \varphi|=O\left(r_{ \pm c}^{-1 / 2}\right) & \left(r_{ \pm c}=\sqrt{(x \mp c)^{2}+y^{2}} \rightarrow 0\right) \\
|\nabla \varphi|=O\left(r_{ \pm d}^{-3 / 2}\right) & \left(r_{ \pm d}=\sqrt{(x \mp d)^{2}+y^{2}} \rightarrow 0\right) .
\end{array}
$$

The asymptotic behaviours given by (25) and (26) are needed to determine the eigensolutions of the problem $(20)-(24)$. Note that the Landau notation also allows weaker singularities for $\nabla \varphi$ at the inner contact point in (25). Indeed, 
it will be shown that $|\nabla \varphi| \sim r_{ \pm c}^{1 / 2}$ there. Equations $(20)-(24)$ provide a mixed boundary-value problem for the complex acceleration $\omega_{0}(z)=\varphi_{x}-i \varphi_{y}$, $z=x+i y$. This function is analytic in $y<0$ and decays in the far field. The problem (20) - (24) can be reformulated as a Dirichlet problem by multiplying the function $\omega_{0}(z)$ by the complex-valued characteristic function

$$
g(z)=\sqrt{\left(z^{2}-c^{2}\right)\left(z^{2}-d^{2}\right)} .
$$

This function is defined in the $z$-plane with cuts along the intervals $(-d,-c)$ and $(c, d)$ of the real axis. Using the theory of boundary problems for analytic functions we obtain the following solution for $\omega_{0}(z)[3]$ :

$$
\begin{aligned}
\omega_{0}(z) & =i \ddot{h}\left(1-\sqrt{\frac{z^{2}-C}{z^{2}-D}}-\frac{1}{2} G(t) \frac{\sqrt{z^{2}-C}}{\left(z^{2}-D\right)^{3 / 2}}\right), \\
G(t) & =\frac{2}{E(q)}\left(\frac{p_{c}}{\varrho \ddot{h}} \sqrt{D}+C K(q)\right)-2 D, \\
q & =\sqrt{1-C / D},
\end{aligned}
$$

where $C=c^{2}, D=d^{2}$. The functions $K(q)$ and $E(q)$ are the complete elliptic integrals of the first and second kind. Details of the calculations to obtain (28)-(30) are presented in the Appendix. It is shown there that eigensolutions of (20)-(26) with square-root singularities at $z= \pm c$ are not permitted in (28) due to the inequalities (15) and (16). It follows that the vertical acceleration of the fluid at the separation point and of the body are equal. It can be further shown that the curvature of the free surface and of the body are equal at the separation point (see [19]). Separation points satisfying such a behaviour are also known as Brillouin points [1].

\subsection{Velocity potential}

We solve the hydrodynamic problem in terms of the complex velocity $\omega_{1}(z)=$ $\phi_{x}-i \phi_{y}$, given by equations (5) - (9) where we rewrite the boundary conditions (7) and (8) by integrating them in time and differentiating with respect to $x$ :

$$
\begin{aligned}
& \phi_{x}=0 \quad(y=0,|x|>d), \\
& \phi_{x}=u(x) \quad(y=0,|x|<c),
\end{aligned}
$$

where $u(x)$ is an undetermined function due to time integration and must be determined as part of the solution. Since the flow is symmetric with respect to $x=0$ we know that $u(x)$ is an odd function, $u(-x)=-u(x)$, and, in particular, $u(0)=0$. The problem (5), (6), (9), (31), (32) can be reformulated as a Dirichlet problem if we consider the function $\left(\omega_{1}(z)-i \dot{h}\right) g(z), z=x+i y$ where $g(z)$ is defined in $(27)$. Then the complex velocity is given by

$$
\omega_{1}(z)=i\left(\dot{h}+\frac{\mathcal{K}\left(z^{2}, t\right)+\dot{h}\left(\frac{1}{2} C+\frac{1}{2} D-z^{2}\right)+A(t)}{\sqrt{\left(z^{2}-C\right)\left(z^{2}-D\right)}}\right),
$$




$$
\mathcal{K}(z, t)=-\frac{1}{\pi} \int_{0}^{C} \frac{\sqrt{(C-\xi)(D-\xi)}}{\xi-z} U(\xi) \mathrm{d} \xi,
$$

where $U(x)=u(\sqrt{x})$. Note that the solution (33) involves an eigensolution with a coefficient $A(t)$ which is an unknown function of time. Since $\dot{\omega}_{1}=\omega_{0}$ we obtain the following equation by comparing the far-field behaviour of $\omega_{0}$ and $\omega_{1}$ in $(28)$ and (33):

$$
\dot{A}=\frac{\ddot{h}}{2}(C-D-G),
$$

where $G$ is defined in (29). Equation (35) is used later to determine $A(t)$. Furthermore, we compare the behaviour of $\omega_{0}(z)$ and $\omega_{1}(z)$ at $z=d$ and obtain

$$
(D-C)\left(\frac{1}{2} \dot{h}-\frac{G \ddot{h}}{\dot{D}}\right)=A+\frac{1}{\pi} \int_{0}^{C} \sqrt{\frac{C-\xi}{D-\xi}} U(\xi) \mathrm{d} \xi .
$$

We will use equation (36) later to determine the function $C(t)$. Kutta's condition (14) implies that $\omega_{1}(z)$ is not singular at $z= \pm c$. It follows from (33) that

$$
\int_{0}^{C} \sqrt{\frac{D-\xi}{C-\xi}} U(\xi) \mathrm{d} \xi=-\frac{\pi}{2}(D-C) \dot{h}-\pi A
$$

which is a weak-singular Volterra integral equation of the first kind for $U$. Note that the equations (35)-(37) do not contain the body shape, $y=f(x)$. The body shape is accounted for in the next section where we consider the displacement potential of the flow problem.

\subsection{Displacement potential}

To employ Wagner's condition (11), we need to know the free-surface elevation $\eta(x, t)$. We could evaluate $\eta(x, t)$ by using the solution of the hydrodynamic problem from subsection 4.2 and integrating with respect to $t$ the kinematic boundary condition (12). However, it is convenient to evaluate the kinematics of the free surface directly by introducing the displacement potential

$$
\Phi(x, y, t)=\int_{0}^{t} \phi(x, y, \tau) \mathrm{d} \tau \quad(y \leq 0)
$$

and reformulating the hydrodynamic problem (5) - (9) with respect to this potential (see [6]). We derive from (5)-(14) that the free surface elevation is given by $[19]$

$$
\eta(x, t)=\Phi_{y}(x, 0, t) \quad(|x|<c \text { and }|x|<d),
$$


where the displacement potential is given by the mixed boundary-value problem

$$
\begin{aligned}
\nabla^{2} \Phi & =0 \quad(y<0), \\
\Phi_{y} & =f(x)-h(t) \quad(y=0, c<|x|<d), \\
\Phi_{x} & =0 \quad(y=0,|x|>d), \\
\Phi_{x} & =t u(x)+s(x) \quad(y=0,|x|<c), \\
\Phi & =O\left(r^{-1}\right) \quad\left(r=\sqrt{x^{2}+y^{2}} \rightarrow \infty\right) .
\end{aligned}
$$

The function $s(x)$ is an undetermined term due to time integration. Since $\Phi_{x}(x, 0, t)$ and $u(x)$ are odd functions of $x, s(x)$ is also odd and so $s(0)=0$. We seek the solution $\Phi(x, y, t)$ of the problem (40) - (44) which is bounded together with its first derivatives $\Phi_{x}$ and $\Phi_{y}$ on $y \leq 0$. The derivatives $\Phi_{x}(x, y, t)$ and $\Phi_{y}(x, y, t)$ provide the components of displacement of a liquid particle.

The boundary-value problem $(40)-(44)$ for $\Phi(x, y, t)$ is similar to the problem (5), (6), (9), (31), (32) for $\phi(x, y, t)$. By using the same techniques, we find the complex displacement potential $\omega_{2}(z)=\Phi_{x}-i \Phi_{y}$ in the form

$$
\begin{aligned}
\omega_{2}(z)= & i\left(h+\frac{\mathcal{M}\left(z^{2}, t\right)+h\left(\frac{1}{2} C+\frac{1}{2} D-z^{2}\right)+B(t)}{\sqrt{\left(z^{2}-C\right)\left(z^{2}-D\right)}}\right), \\
\mathcal{M}(z, t)= & -\frac{1}{\pi} \int_{0}^{C} \frac{\sqrt{(C-\xi)(D-\xi)}}{\xi-z}(t U(\xi)+S(\xi)) \mathrm{d} \xi \\
& -\frac{1}{\pi} \int_{C}^{D} \frac{\sqrt{(\xi-C)(D-\xi)}}{\xi-z} F(\xi) \mathrm{d} \xi,
\end{aligned}
$$

where $F(x)=f(\sqrt{x})$ and $S(x)=s(\sqrt{x})$. The solution $\omega_{2}(z)$ involves an eigenfunction with a coefficient $B(t)$, which is an unknown function of time. Since $\dot{\omega}_{2}=\omega_{1}$ we obtain the following equation for $B(t)$ by comparing the far-field behaviour of $\omega_{1}$ and $\omega_{2}$ in (33) and (45):

$$
\dot{B}=A \text {. }
$$

Since $\omega_{2}(z)$ is bounded at $z= \pm c$ and $z= \pm d$ we obtain the following two relations:

$$
\begin{aligned}
\begin{array}{l}
\int_{0}^{C} \sqrt{\frac{C-\xi}{D-\xi}}(t U(\xi)+S(\xi)) \mathrm{d} \xi=-\int_{C}^{D} \\
\sqrt{\frac{\xi-C}{D-\xi}} F(\xi) \mathrm{d} \xi \\
+\frac{\pi}{2}(D-C) h-\pi B
\end{array} \\
\begin{aligned}
\int_{0}^{C} \sqrt{\frac{D-\xi}{C-\xi}} S(\xi) \mathrm{d} \xi= & \int_{C}^{D} \sqrt{\frac{D-\xi}{\xi-C}} F(\xi) \mathrm{d} \xi \\
& -\frac{\pi}{2}(D-C)(h-t \dot{h})-\pi(B-t A) .
\end{aligned}
\end{aligned}
$$

Note that we used (37) to arrive at (49). This integral equation is of the same structure as (37) and will be used later to determine the cavity function $S(x)$. 
We find an ordinary differential equation for $D(t)$ by differentiating equation (48) with respect to time. Together with equations (35) and (47) we find a system of differential equations for $A, B$ and $D$. The time $t_{0}$ and the initial conditions for $D, A$ and $B$ at $t=t_{0}$ are given by equations (17), (19) and by (see section 4.5)

$$
A=-\frac{1}{2} D \dot{h}, \quad B=\frac{1}{\pi} \int_{0}^{D} \sqrt{\frac{D-\xi}{\xi}} F(\xi) \mathrm{d} \xi-\frac{1}{2} D h \quad\left(t=t_{0}\right) .
$$

The functions $U(x)$ and $S(x)$ are obtained by solving the integral equations (37) and (49). We determine $C$ by equation (36).

\subsection{Hydrodynamic pressure and force}

The pressure $p(x, 0, t)$ along the contact region is determined by integration of $p_{x}(x, 0, t)$ with respect to $x$ where the constant of integration is given by $p(c, 0, t)=p_{c}$. The function $p_{x}(x, 0, t)$ is obtained from the real part of $\omega_{0}(z)$ in equation (28) and the linearised Bernoulli's equation (4):

$$
p_{x}(x, 0, t)=\varrho \ddot{h}\left(-\sqrt{\frac{x^{2}-C}{D-x^{2}}}+\frac{G}{2} \frac{\sqrt{x^{2}-C}}{\left(D-x^{2}\right)^{3 / 2}}\right),
$$

where $G$ is defined in (29). The pressure in the cavitation stage, given by (51), only implicitly depends on the vertical speed of the body, $\dot{h}$, since $C$ depends on $\dot{h}$ in (36). This contrasts with the pressure (18) in the Wagner stage where $\dot{h}$ explicitly appears.

The total vertical force acting on the body, including the contribution from the cavity region, is given by

$$
L(t)=\int_{R_{w} \cup R_{c}} p(x, 0, t) \mathrm{d} x, \quad L(t)=\frac{\pi}{2} \varrho \ddot{h}(G+D-C) .
$$

Part of $L(t)$ is the vertical hydrodynamic force $L_{h}=\int_{R_{w}} p(x, 0, t) \mathrm{d} x$ due to the liquid wetting the body. This is given by $L_{h}(t)=L(t)-2 \sqrt{C} p_{c}$.

\subsection{Asymptotic analysis of early cavity growth}

In this section, we investigate the flow at the initial stage of the cavity formation, $t-t_{0} \ll 1$, for any $p_{c}$ and confirm the asymptotic results for $p_{c}=0$ from [10]. Such an analysis is helpful to start the numerical calculations for $t>t_{0}$. We assume that the horizontal size of the cavity, $c(t)$, is continuous in time starting from a single point, $c\left(t_{0}\right)=0$. Furthermore, we consider only motions of the body where the body displacement $h(t)$ is continuous together with its first and second derivatives. Otherwise, the pressure does not evolve continuously in time. Such a discontinuity might cause an instantaneous detachment 
of the fluid from a non-zero length of the body surface for which we start with $c\left(t_{0}\right)>0$. Note that jumps or singularities of the higher time derivatives of $h$ starting from the third one are allowed. The asymptotic solution of $(35)-(37)$, (47)-(49) is sought by using the expansion procedure about $t=t_{0}$ for $A(t)$, $B(t), d(t)$ and about $x=0$ for $u(x)$ :

$$
\begin{aligned}
& A(t)=A_{0}+A_{1} \Delta t+O\left(\Delta t^{2}\right), \\
& B(t)=B_{0}+B_{1} \Delta t+\frac{1}{2} B_{2} \Delta t^{2}+O\left(\Delta t^{3}\right), \\
& d(t)=d_{0}+d_{1} \Delta t+\frac{1}{2} d_{2} \Delta t^{2}+O\left(\Delta t^{2}\right), \\
& u(x)=u_{1} x+O\left(x^{3}\right)
\end{aligned}
$$

where $\Delta t=t-t_{0}$. Note that $A_{0}=B_{1}$ and $A_{1}=B_{2}$ due to equation (47). The form of the expansion of $s(x)$ depends on the shape of the body. However, we know that $s(0)=0$, since $s(x)$ is an odd function. The coefficients $A_{i}, B_{i}, d_{i}$ and $u_{i}$ are obtained by substituting expansions (53)-(56) into equations (35)(37), (48), (49). The leading order equations of (35)-(37), (48), (49) together with equations (17) and (19) give us

$$
\begin{aligned}
& A_{0}=B_{1}=-\frac{1}{2} d_{0}^{2} \dot{h}\left(t_{0}\right), \quad A_{1}=B_{2}=\frac{1}{2} d_{0}^{2} \ddot{h}\left(t_{0}\right)-\frac{p_{c}}{\varrho} d_{0}, \\
& B_{0}=\frac{1}{\pi} \int_{0}^{d_{0}^{2}} \sqrt{\frac{d_{0}^{2}-\xi}{\xi}} F(\xi) \mathrm{d} \xi-\frac{1}{2} d_{0}^{2} h\left(t_{0}\right), \\
& d_{0}=d\left(t_{0}^{-}\right), \quad d_{1}=\dot{d}\left(t_{0}^{-}\right) .
\end{aligned}
$$

It follows from the second order of (37) that

$$
u_{1}=\frac{\dot{h}\left(t_{0}\right)}{d_{0}} .
$$

The function $s(x)$ is determined by the second order of (49) and its asymptotic form depends on the shape of the body, $y=f(x)$. Here we only give the asymptotic expansions of $s(x)$ for the impact of a parabola, $f(x)=x^{2} /(2 R)$, where $R$ is the curvature radius of the bottom of the body, and for the impact of a wedge, $f(x)=\varepsilon|x|$, where $\varepsilon$ is the deadrise slope of the wedge. For the impact of a parabola we have

$$
s(x)=\left(\frac{1}{d_{0}}\left(h\left(t_{0}\right)-t_{0} \dot{h}\left(t_{0}\right)\right)+\frac{d_{0}}{4 R}\right) x+O\left(x^{3}\right) .
$$

For the impact of a wedge we have

$$
s(x)=\left(\frac{1}{d_{0}}\left(h\left(t_{0}\right)-t_{0} \dot{h}\left(t_{0}\right)\right)-\frac{2 \varepsilon}{\pi}-\frac{2 \varepsilon}{\pi} \log \left(\frac{x}{2 d_{0}}\right)\right) x+O\left(x^{3}\right) .
$$

An asymptotic behaviour of $c(t)$ at $t=t_{0}$ can be found using the following expansion of $\Delta t$ about $c=0$ :

$$
\Delta t(c)=T c^{2} \log (k c)+O\left(c^{4} \log ^{2}(c)\right) .
$$


It follows from the second order of (36) that

$$
T=\frac{\frac{1}{2} \frac{p_{c}}{\varrho}-\ddot{h}\left(t_{0}\right) d_{0}}{d_{0}^{2}\left(\dot{h}\left(t_{0}\right) d_{2}+2 \ddot{h}\left(t_{0}\right) d_{1}+\dddot{h}\left(t_{0}^{+}\right) d_{0}\right)}, \quad k=\frac{1}{4 d_{0} \sqrt{e}} .
$$

Note that we needed that $\dot{h}\left(t_{0}^{-}\right)=\dot{h}\left(t_{0}^{+}\right)$and $\ddot{h}\left(t_{0}^{-}\right)=\ddot{h}\left(t_{0}^{+}\right)$to derive equations in (64) but it is allowed that $\dddot{h}\left(t_{0}^{-}\right) \neq \dddot{h}\left(t_{0}^{+}\right)$as long as $\left|\dddot{h}\left(t_{0}^{+}\right)\right|<\infty$ and $\left|\dddot{h}\left(t_{0}^{+}\right)\right|<\infty$. Since the asymptotic behaviour of $c$ given by equations (63) and (64) depends on the third time-derivative of $h$, the evolution of the inner contact point is very sensitive to the motion of the body.

The first time-derivative of equation (48) at $t=t_{0}$ is together with (57)(59) and the derivative of (17) an identity. This confirms that the assumption $c\left(t_{0}\right)=0$ is correct. The second time-derivative of equation (48), equations (57)-(59) and the first two derivatives of (17) confirm that $d_{2}=\ddot{d}\left(t_{0}^{-}\right)$. Hence, the outer contact points evolve very smoothly at the transition from the Wagner stage to the cavitation stage. This result supports the correctness of our cavitation modelling.

The thickness of the cavity at $x=0$ is defined as $\delta(t)=f(0)-h(t)-\eta(0, t)$. The vertical acceleration of the free surface at $x=0, \eta_{t t}(0, t)$, is given by the imaginary part of $\omega_{0}(z)$ in equation $(28)$ so that the second derivative of $\delta(t)$ is given by

$$
\ddot{\delta}(t)=\frac{\ddot{c h}}{d^{3}}\left(\frac{1}{2} G-d^{2}\right) .
$$

We obtain the following initial thickness of the cavity by integrating (65) twice in time subject to the initial conditions $\dot{\delta}\left(t_{0}\right)=0$ and $\delta\left(t_{0}\right)=0$ and by using (63):

$$
\begin{aligned}
\delta(t) & =\mu c^{5}\left(\log ^{2}\left(\frac{c}{4 d_{0}}\right)-\frac{11}{15} \log \left(\frac{c}{4 d_{0}}\right)+\frac{11}{75}\right)+O\left(c^{7} \log ^{3}(c)\right), \\
\mu & =\frac{8\left(\frac{1}{2} \frac{p_{c}}{\varrho}-\ddot{h}\left(t_{0}\right) d_{0}\right)^{3}}{15\left(\dot{h}\left(t_{0}\right) d_{2}+2 \ddot{h}\left(t_{0}\right) d_{1}+\dddot{h}\left(t_{0}^{+}\right) d_{0}\right)^{2} d_{0}^{6}} .
\end{aligned}
$$

Equation (66) shows that the thickness of the cavity is strongly dependent on the body deceleration at the instant of cavity formation but is small at the initial stage. For $p_{c}=0$ equation (63) and (66) are identical to the ones in [10], though the methods for solving the problem are different. It will be shown in the next section, how the present asymptotic analysis helps us to start computations during the cavitation stage.

\section{Numerical results}

In the cavitation stage we investigate the interaction between the body, the fluid free surface and the cavity numerically. The numerical scheme involves three stages at each time step. In a first stage we integrate equations (35) and 
(47) and the time-derivative of equation (48) to obtain $A, B$ and $D$ for the next time step. For integration, we use an explicit modified Euler's method where we set $C$ to its value computed in the previous time step. The values $U(\xi)$ and $S(\xi)$ in the time-derivative of equation (48) are approximated by linear splines and were already computed in the previous time steps. Initial conditions for the resulting system of ordinary differential equations system are given by (50). In the second stage we find the position of the separation point $C$ from equation (36) by a secant method. However, for the first time step we experienced convergence problems with the secant method. We avoided these difficulties, by approximating the value of $C$ in the first time step only, by exploiting the asymptotic expansion (63). In the third stage we solve the integral equations (37) and (49) with respect to $U(C)$ and $S(C)$.

Numerical results are shown in Figures 2-10 for the impact of parabolas and wedges moving vertically with constant vertical deceleration $a$. Hence, the penetration depth is given by $h(t)=t\left(V-\frac{a}{2} t\right)$, where the constant $V$ is the initial vertical speed of the body. We compare the present cavitation model with the Wagner model in which cavitation is not included.

\subsection{Impact of a parabola}

The shape of a parabola is given by $f(x)=x^{2} /(2 R)$, where $R>0$ is the radius of curvature of the body surface at its lowest point. We are concerned with the initial stage of impact, whose duration is of order $V / a$. Since the penetration depth is of order $V^{2} / a$, the horizontal extent of the wetted region is of order $V \sqrt{R / a}$. Using these scalings, equations (35)-(37), (47)-(49) can be formulated in non-dimensional variables with the non-dimensional cavitation pressure $p_{c}^{*}=p_{c} /(\varrho V \sqrt{a R})$ as the only parameter. For example, the non-dimensional penetration depth is given by $h_{*}\left(t_{*}\right)=t_{*}\left(1-t_{*} / 2\right)$ where the asterisks stand for the variables in non-dimensional form. Note that the Wagner theory can only be used while the penetration depth of the body is much smaller than the extent of the wetted region and so the parameter $V / \sqrt{a R}$ has to be small.

During the Wagner stage, the non-dimensional position of the contact point is given by $d_{*}\left(t_{*}\right)=2 \sqrt{h_{*}\left(t_{*}\right)}$. The hydrodynamic pressure at the lowest point of the parabola is given by $p_{0}^{*}=\left(1-4 h_{*}\right) / \sqrt{h_{*}}$. We obtain $\min \left(p_{0}^{*}\right)=-\sqrt{2}$ since the maximum penetration depth is $h_{*}=\frac{1}{2}$. Hence, cavitation can only occur if the following inequality is satisfied

$$
\frac{\left|p_{c}\right|}{\varrho V \sqrt{R a}}<\sqrt{2},
$$

where atmospheric pressure is set to zero. In water, a cavity at vapour pressure $p_{c}=-101.3 \mathrm{kPa}+2.3 \mathrm{kPa}=-99.0 \mathrm{kPa}$ (see [7]) develops only if the parameters $V, R$ and $a$ satisfy $V \sqrt{R a}>70 \mathrm{~m}^{2} \mathrm{~s}^{-2}$.

The computations for the cavitation stage were performed with non-dimensional time step 0.001 . The positions of the body and the free surface at several 


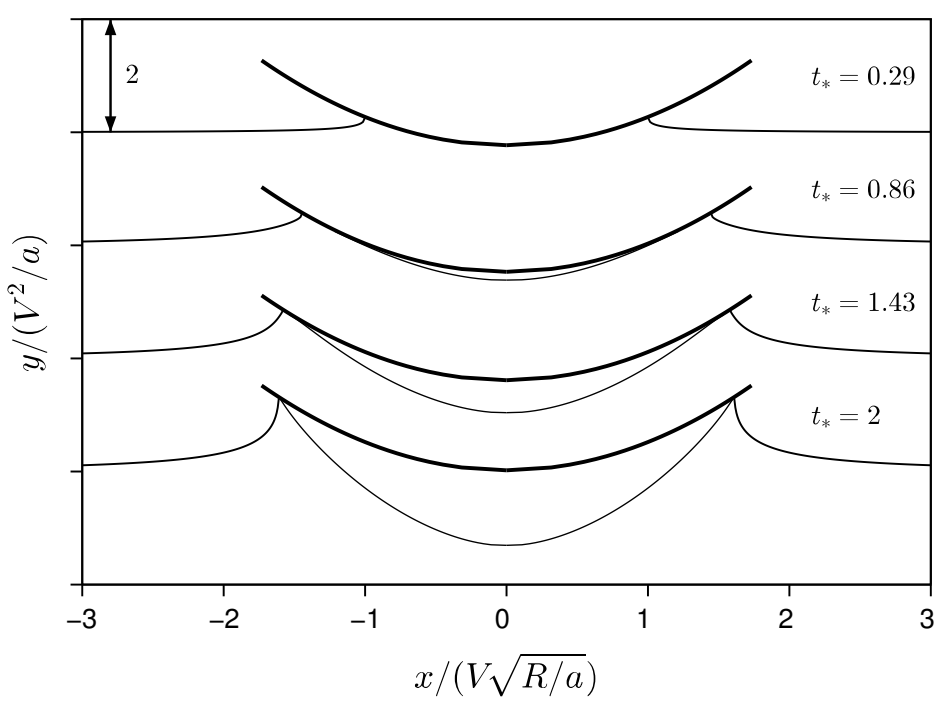

Fig. 2 Impact of the parabolic body with $p_{c}=0$ : The body positions (thick lines) and the free-surface elevations (thin lines) are shown at different time instants where $t_{*}=t a / V$ is the non-dimensional time. Note the different scalings of $x$ - and $y$-axes.

times are shown in Figure 2 for $p_{c}^{*}=0$. The size of the cavity increases in time with the wetted regions on the body surface being very small at $t_{*}=2$. In our model, computations show that the fluid does not detach from the body but the body is connected with the fluid at the two turnover regions even at $t_{*}=2$. Note that the body moves upwards when $t_{*}>1$, which corresponds to the exit stage. The position of the body at $t_{*}=2$ is the same as at $t_{*}=0$. For $t_{*}>2$ the wetted regions continue to shrink and our model becomes not reliable.

The motions of the outer and inner contact points, $x_{*}=c_{*}\left(t_{*}\right)$ and $x_{*}=$ $d_{*}\left(t_{*}\right)$, are shown for $p_{c}^{*}=0$ in Figure 3 and are compared with the motions of the point of zero pressure, $x_{*}=\hat{c}_{*}\left(t_{*}\right), p_{*}\left(\hat{c}_{*}, 0, t_{*}\right)=0$, and the contact point in the model without a cavity. Note that for $p_{c}^{*}=0$ the cavitation stage starts at $t_{*}=1-\sqrt{2} / 2 \approx 0.29$. The positions of the inner contact points $x_{*}= \pm c_{*}\left(t_{*}\right)$ and the zero-pressure points $x_{*}= \pm \hat{c}_{*}\left(t_{*}\right)$ differ significantly. In the model without a cavity, the zero pressure points meet the contact points at $t_{*}=1$, when the body stops penetrating the fluid. At this time instant the speeds of the contact points are zero. It has been shown in [6] that the Wagner model breaks down when the Wagner contact points stop advancing. An extension of the Wagner model from $t_{*}=1$ onwards, a so-called exit model, has been under intensive research (see e.g. [11]). Figure 3 shows for the model with a cavity, that the speeds of the inner and outer contact points decrease and their positions approach the asymptotic value of $x_{*} \approx \pm 1.6$. However, 


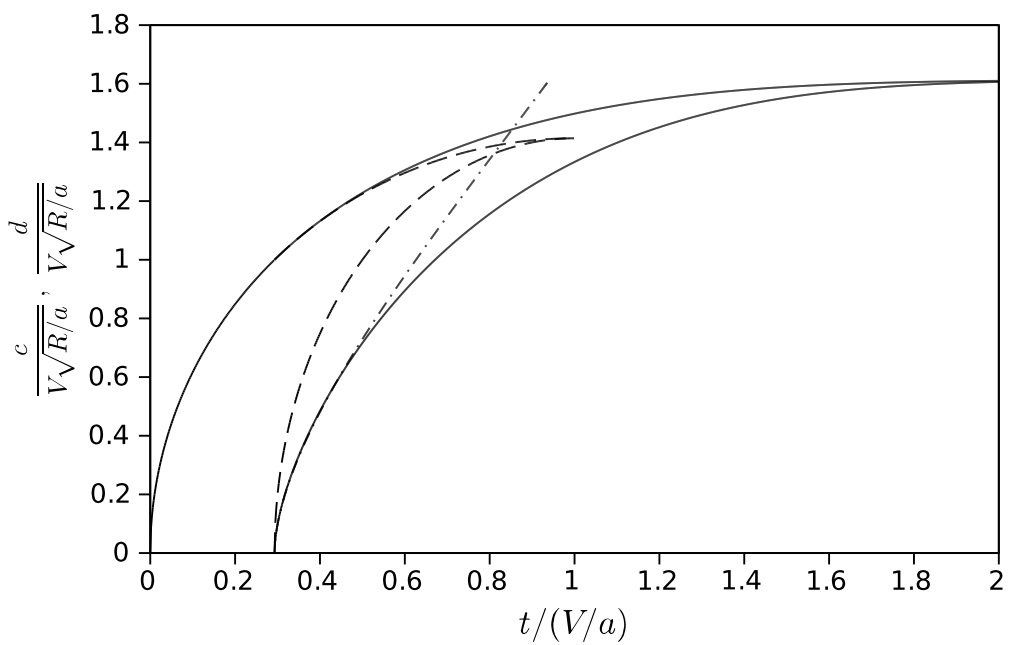

Fig. 3 Motion of the inner and outer contact points for $p_{c}^{*}=0$ are shown as solid lines for the impact of a parabola. The position of the contact point and the position on the body surface where $p_{*}\left(x_{*}, 0, t_{*}\right)=0$ in the original Wagner model (no cavity) are shown as dashed lines. The dash-dot line describes the asymptotic behaviour of $c_{*}$ at the initial stage of cavity formation given by equation (63) where $k_{*}=1 /(4 \sqrt{e})$ and $T_{*}=-\sqrt{2} / 8$.

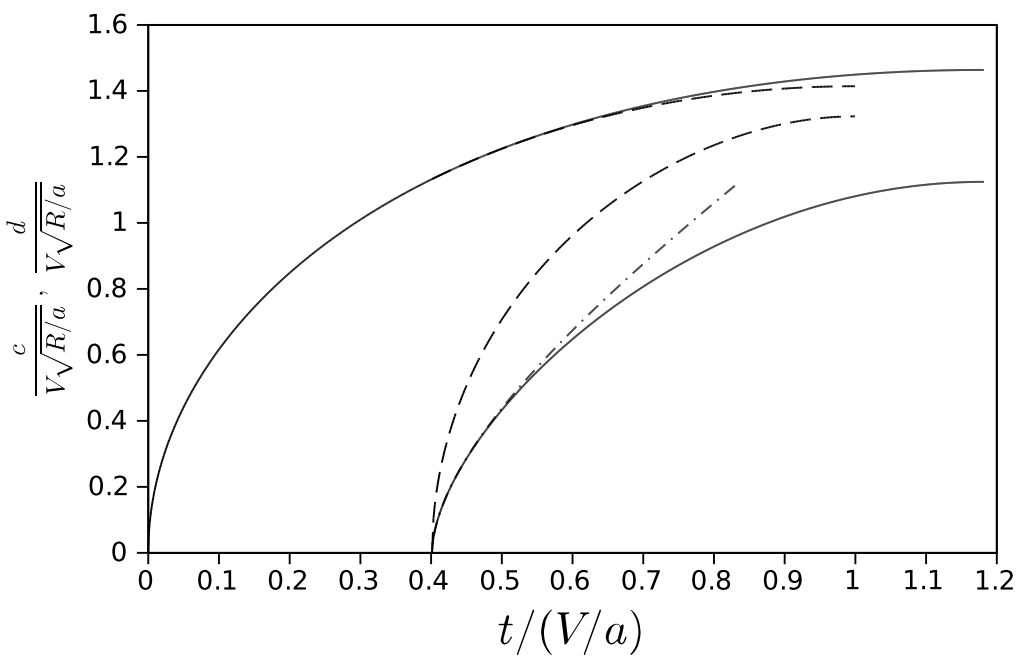

Fig. 4 Motion of the inner and outer contact points for $p_{c}^{*}=-0.5$ are shown as solid lines for the impact of a parabola. The position of the contact point and the position on the body surface where $p_{*}\left(x_{*}, 0, t_{*}\right)=-0.5$ in the original Wagner model (no cavity) are shown as dashed lines. The dash-dot line shows the asymptotic behaviour of $c_{*}$ at the initial stage of cavity formation given by equation (63). 
within the present model we could not find a time instant when the inner and outer contact points meet each other or the velocities of the contact points become zero. The structure of the inner solution at the outer contact points when the size of the wetted region becomes small has not been investigated in the present paper but might give a deeper insight into this exit problem. Note that such a behaviour of the contact points only occurs if $p_{c}^{*}=0$. For $p_{c}^{*}<0$ the cavitation stage starts later and the speeds of the outer contact points decrease to zero in finite time. Figure 4 shows that the cavitation stage starts at $t_{0}^{*} \approx 0.40$ for $p_{c}^{*}=-0.5$ and we have to stop our computation at $t_{*} \approx 1.18$ when $\dot{d}_{*}\left(t_{*}\right)=0$, because our model is only valid while $\dot{d}_{*}>0$. A non-trivial extension of the present model which is capable to describe the flow after $\dot{d}_{*}$ becomes zero is needed. Such an extension is not further discussed in this paper. It follows from equation (36) that the final position of the inner contact point, $x_{*}=c_{*}$, when $\dot{d}_{*}=0$, is given in terms of $d_{*}$ by

$$
c_{*}^{2} K(q)-d_{*}^{2} E(q)=p_{c}^{*} d_{*}, \quad q=\sqrt{1-c_{*}^{2} / d_{*}^{2}} .
$$

For $p_{c}^{*}=-0.5$, the final positions of the contact points $c_{*} \approx 1.12$ and $d_{*} \approx 1.46$ satisfy this equation. For our first example with $p_{c}^{*}=0$ we formally obtain from equation (69) that $c_{*}\left(t_{*}\right)=d_{*}\left(t_{*}\right)$. This confirms that the inner contact points approach the outer contact points for increasing time. It is also remarkable that in all cases considered in this paper the speed of the inner contact points becomes zero when $\dot{d}_{*}=0$.

Figure 5 shows the nondimensional vertical force $L_{*}\left(t_{*}\right)=L /\left(\varrho R V^{2}\right)$ (see eq. (52)) acting on the parabola for $p_{c}^{*}=0$ and $p_{c}^{*}=-0.5$, and for the model without a cavity. The force is initially $L_{*}(0)=2 \pi$ and decays quickly due to the deceleration of the body. For $p_{c}^{*}=0$ the hydrodynamic force is always positive and decays to zero as $t_{*} \rightarrow \infty$. For $p_{c}^{*}=-0.5$ the hydrodynamic force becomes negative. Note that the hydrodynamic force $L_{h}^{*}$ acting on the fluid-body interface is larger than $L_{*}$ since the pressure in the cavity region is negative. For the model without a cavity, negative forces on the body are maximal, so that we obtain the lowest hydrodynamic loads.

Pressure distributions of both models are presented along the wetted part of the parabola and fluid-cavity interface in Figure 6 for $p_{c}^{*}=-0.5$. At $t_{*}=$ $t_{0}^{*}$ these pressure distributions are identical. For time $t_{*}>t_{0}^{*}$ the pressure distributions signifcantly differ. The pressure is constant along the fluid-cavity interface whereas the pressure is far below cavitation pressure in the model without a cavity. Note the pressure inverse-square-root singularities at the outer contact points $x_{*}= \pm d_{*}$. Close to $x_{*}= \pm d_{*}$, the differences between the pressures predicted by the models are small.

The non-dimensional thickness of the cavity, $\delta_{*}\left(t_{*}\right)=\delta(t) /\left(V / a^{2}\right)$, at $x_{*}=$ 0 is shown as a function of time for $p_{c}^{*}=0$ and $p_{c}^{*}=-0.5$ in Figure 7 . It is seen that at the initial stage of cavity formation the cavity is very thin but $\delta_{*}$ quickly grows later on.

Our computations show that the functions $A_{*}\left(t_{*}\right), B_{*}\left(t_{*}\right), c_{*}\left(t_{*}\right), d_{*}\left(t_{*}\right)$ and $\delta_{*}\left(t_{*}\right)$ are well approximated by the asymptotic functions given in section 4.5 (without the higher order terms specified) for at least $0 \leq t_{*}-t_{0}^{*} \leq 0.2$. This 


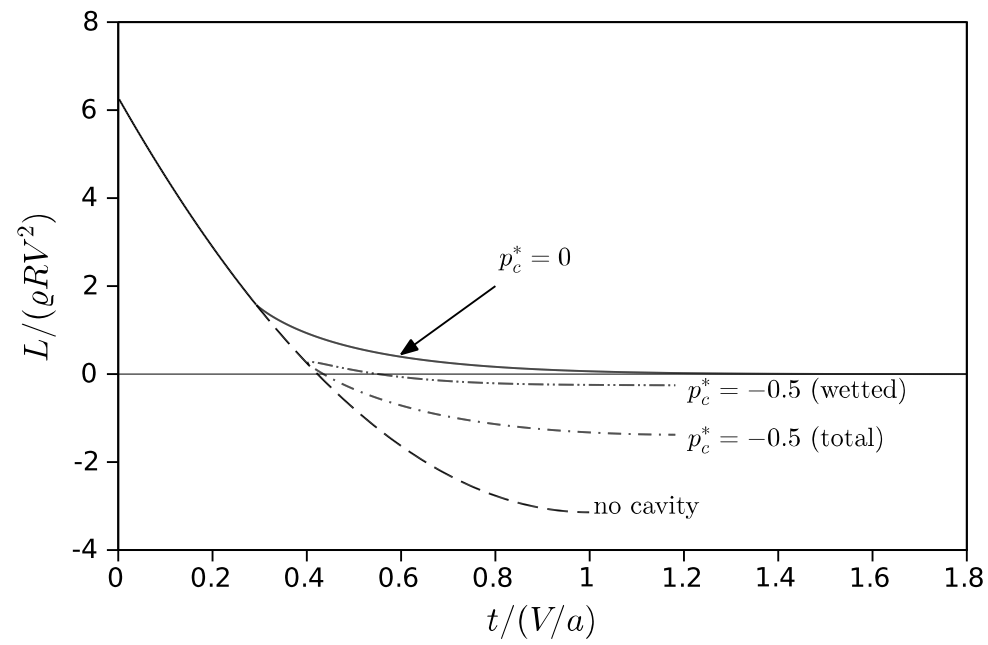

Fig. 5 Non-dimensonal vertical force $L_{*}$ on a parabola as a function of non-dimensional time for $p_{c}^{*}=0$ (solid line) and $p_{c}^{*}=-0.5$ (dash-dot line). The dashed line is the vertical force for the model without a cavity. For $p_{c}^{*}=-0.5$ the hydrodynamic component of the vertical force $L_{h}^{*}=L_{*}-2 c_{*} p_{c}^{*}$ acting on the fluid-body interface $c_{*}<\left|x_{*}\right|<d_{*}$ is plotted as a dash-dot-dot line. Note $t_{*}=1$ is the time of lowest position of the body.

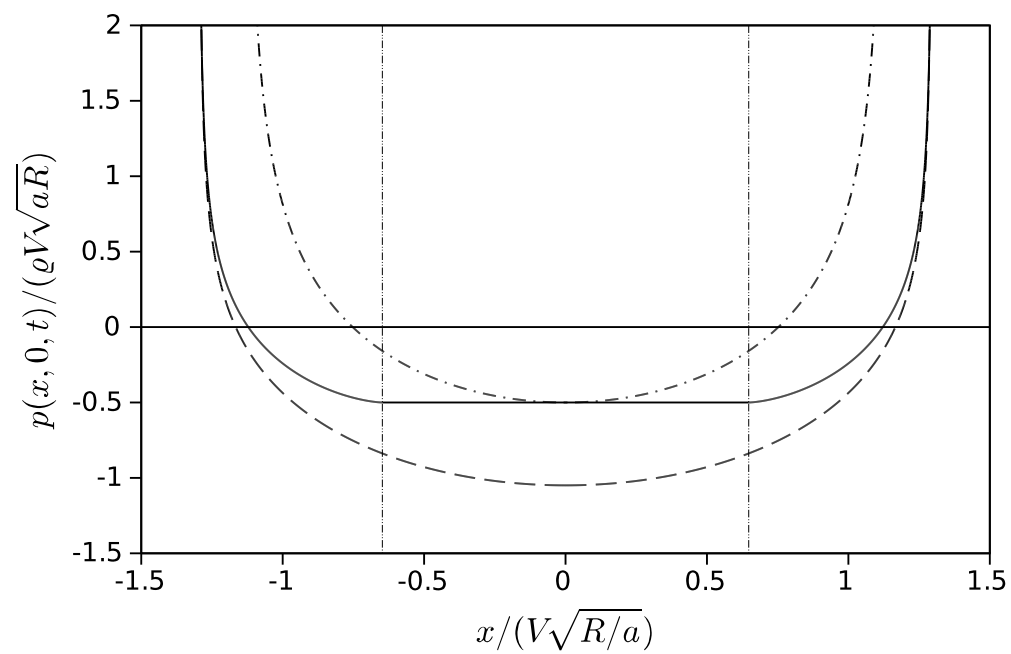

Fig. 6 Hydrodynamic pressure distribution along the parabola at time $t_{*}=0.6$ for the models accounting for a cavity where $p_{c}^{*}=-0.5$ (solid line) and without a cavity (dashed line). The position of the inner contact points are indicated by the thin vertical lines. The pressure distributions of both models are identical at $t_{*}=t_{0}^{*} \approx 0.40$ (dash-dot line). 


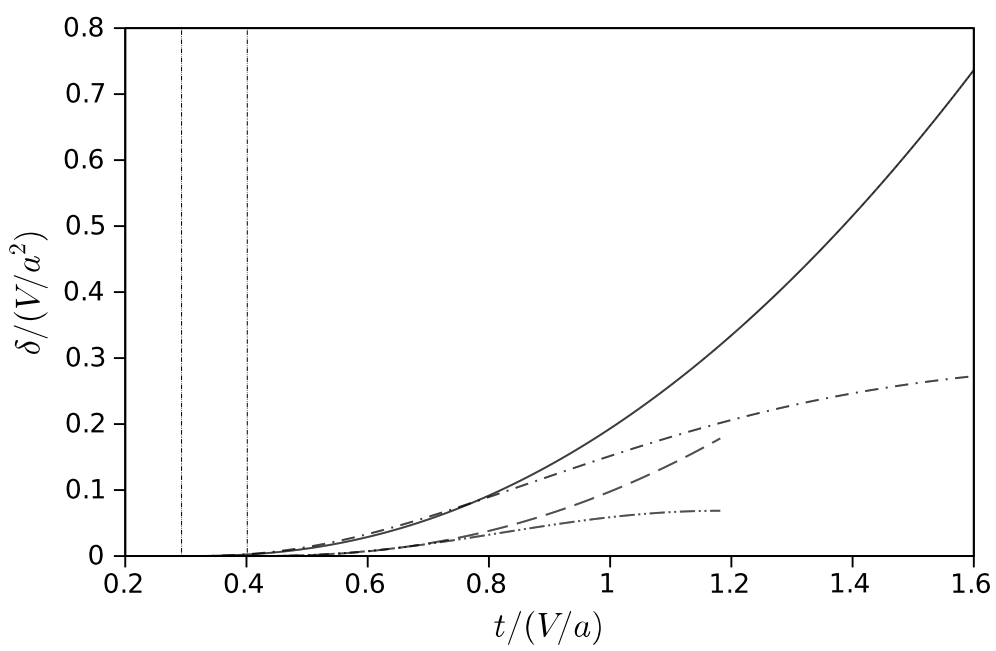

Fig. 7 Thickness of the cavity, $\delta_{*}\left(t_{*}\right)$, at $x=0$ as a function of non-dimensional time $t_{*}=t a / V$ for the impact of a parabola. The solid line is for $p_{c}^{*}=0$ and the dashed line for $p_{c}^{*}=-0.5$. The thin vertical lines indicate the times when the cavitation stages start $\left(t_{0}^{*} \approx 0.3\right.$ for $p_{c}^{*}=0$ and $t_{0}^{*} \approx 0.4$ for $\left.p_{c}^{*}=-0.5\right)$. The dash-dot and the dash-dot-dot lines describe the asymptotic behaviours of $\delta(t)$ for $p_{c}^{*}=0$ and $p_{c}^{*}=-0.5$ given in equation (66) (without higher-order terms).

is demonstrated for $c_{*}\left(t_{*}\right)$ in Figures 3 and 4 and for $\delta_{*}\left(t_{*}\right)$ in Figure 7 . These agreements support the correctness of the results from our computations.

\subsection{Impact of a wedge}

The surface of a wedge is described by the function $f(x)=\varepsilon|x|, \varepsilon \ll 1$, where $\varepsilon$ is the deadrise slope of the wedge. With the scale of the penetration depth being $V^{2} / a$, the scale of the horizontal size of the wetted area is $V^{2} /(\varepsilon a)$. The timescale is given by $V / a$. The equations (35)-(37), (47)-(49) of our model can be formulated in non-dimensional variables with the non-dimensional cavitation pressure $p_{c}^{*}=p_{c} /\left(\varrho V^{2} / \varepsilon\right)$ as the only parameter.

During the Wagner stage, the position of the contact point is given by $d_{*}\left(t_{*}\right)=\frac{\pi}{2} h_{*}\left(t_{*}\right)$. The hydrodynamic pressure at the tip of the wedge is $p_{0}^{*}=$ $\frac{\pi}{2}\left(1-3 h_{*}\right)$. Since the largest penetration depth before the body starts leaving the water is $h_{*}=\frac{1}{2}$, cavitation can only occur if the following inequality is satisfied:

$$
\frac{\varepsilon\left|p_{c}\right|}{\varrho V^{2}}<\frac{\pi}{4},
$$




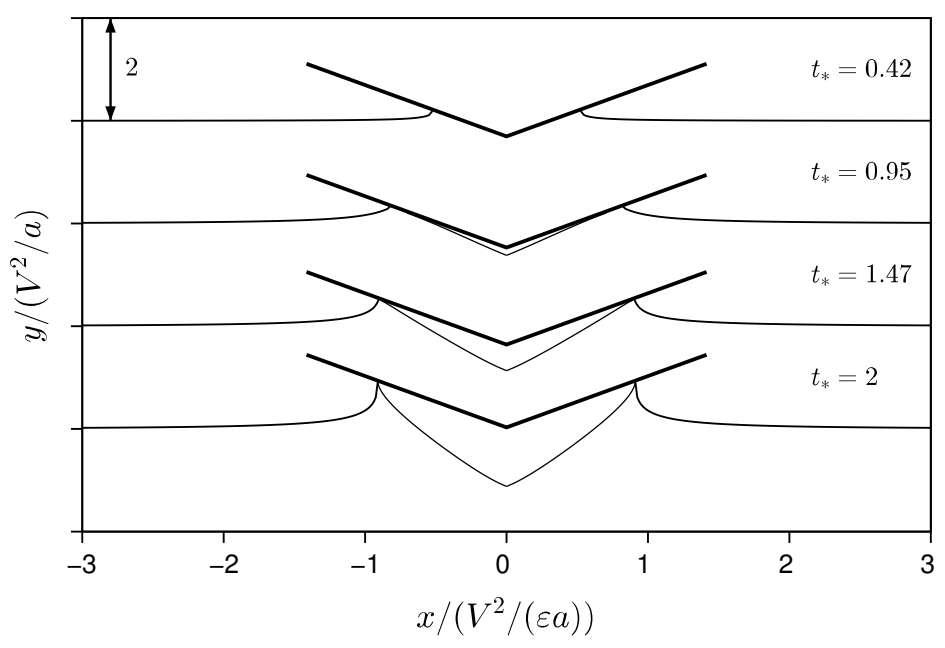

Fig. 8 Impact of a wedge for $p_{c}^{*}=0$ : The time-evolution shows the body positions (thick lines) and the free-surface elevations (thin lines). Note that $t_{*}=t a / V$ and the different scalings of $x$ - and $y$-axes.

where atmospheric pressure is set to zero. Note that condition (70) does not depend on the magnitude of deceleration. In water, a cavity at vapour pressure develops only if $V / \sqrt{\varepsilon}>11.2 \mathrm{~m} \mathrm{~s}^{-1}$.

The computations for the cavitation stage were performed with non-dimensional time step 0.001 . The positions of the wedge and the free surface at several times are shown in Figure 8 for $p_{c}^{*}=0$. These results are similar to the ones for the parabola, except for the kink in the free surface at $x=0$. At $t_{*}=2$ the wetted region of the body is very small. As with the parabola, our computations show that the fluid never completely detaches from the body, instead the body is connected with the fluid at the two turnover regions.

The motions of the inner and outer contact points are shown for $p_{c}^{*}=0$ in Figure 9 and are compared with the motion of the point of zero pressure, $\hat{c}_{*}\left(t_{*}\right), p_{*}\left(\hat{c}_{*}\left(t_{*}\right), 0, t_{*}\right)=0$ and the contact points in the model without a cavity. Due to the deceleration of the body the speeds of the inner and outer contact points decrease and their positions approach the asymptotic value of $x_{*} \approx \pm 0.91$. As with the parabola, we could not find a time when the inner and outer contact points meet each other or the horizontal velocity of one of the contact points becomes zero. For $p_{0}^{*}>0$ we have to stop the computations when $\dot{d}_{*}=0$.

Figure 10 shows the vertical hydrodynamic forces acting on the wedge for $p_{c}^{*}=0$ and $p_{c}^{*}=-0.5$, and for the model without a cavity. In contrast to a parabola, the force is zero initially and decays after reaching a maximum. For $p_{c}^{*}=0$ the hydrodynamic force is always positive and tends to zero as $t_{*} \rightarrow \infty$. 


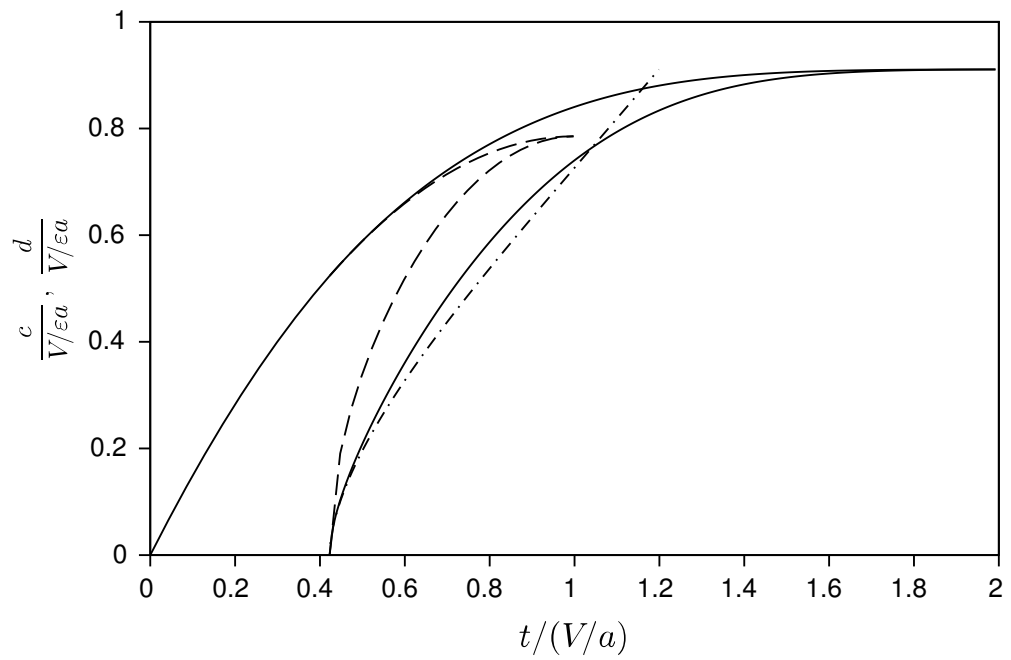

Fig. 9 Motions of the inner and outer contact points for $p_{c}^{*}=0$ are shown as solid lines for the impact of a wedge. The positions of the contact points and the positions of the points on the body surface, where $p_{*}\left(x_{*}, 0, t_{*}\right)=0$ in the model without a cavity, are shown as dashed lines. The dash-dot line describes the asymptotic behaviour of $c_{*}$ at the initial stage of cavity formation described in equation (63) where $k_{*}=3 /(2 \pi \sqrt{e})$ and $T_{*}=-12 /\left(\sqrt{3} \pi^{2}\right)$.

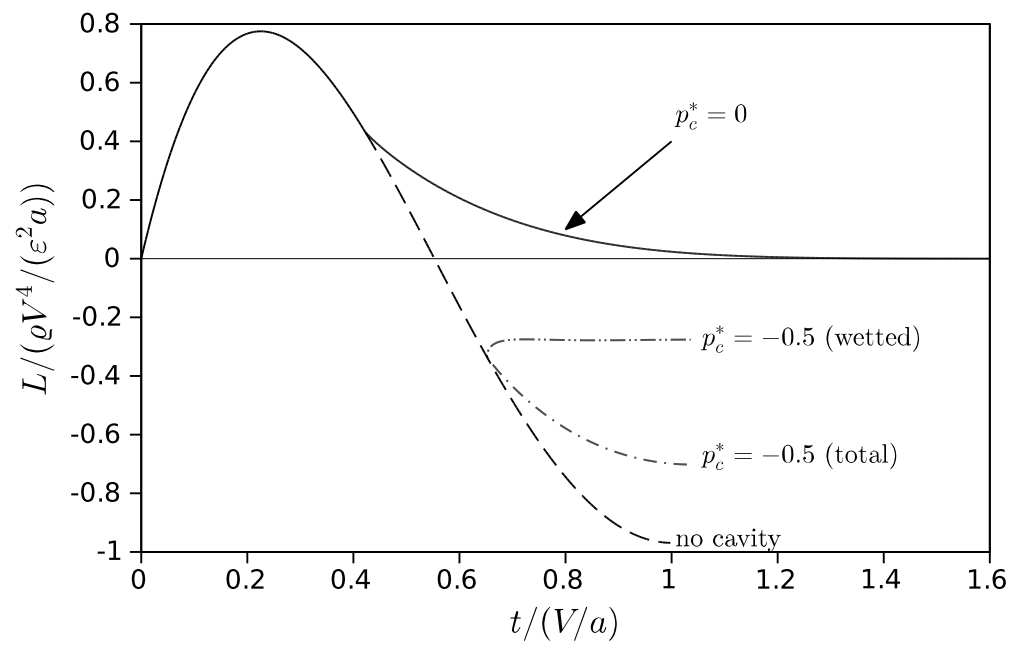

Fig. 10 Non-dimensional vertical force $L_{*}$ acting on a wedge as a function of nondimensional time for $p_{c}^{*}=0$ (solid line) and for $p_{c}^{*}=-0.5$ (dash-dot line). The dashed line corresponds to the vertical force in the model without a cavity. For $p_{c}^{*}=-0.5$ the hydrodynamic component of the vertical force, $L_{h}^{*}=L_{*}-2 c_{*} p_{c}^{*}$, is plotted as a dash-dot-dot line. Note that $t_{*}=1$ is the time of the lowest position of the body. 


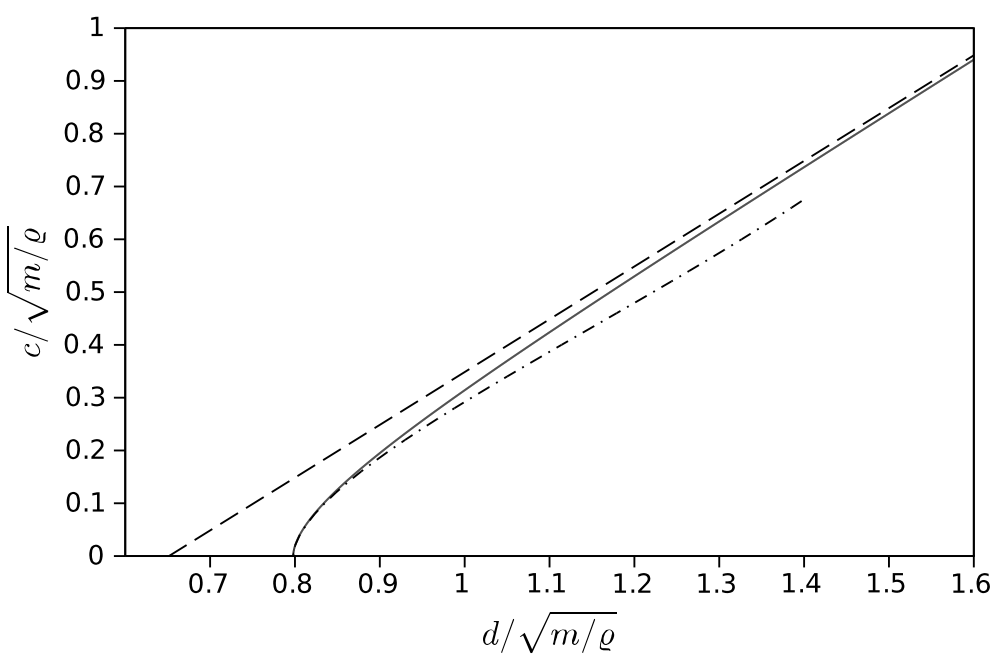

Fig. 11 Plot of $c_{*}=c \sqrt{\varrho / m}$ as a function of $d_{*}=d \sqrt{\varrho / m}$ (solid line) for a freely moving symmetric body. The asymptotic behaviour at $c_{*}=0$ is given by $d_{*}=\sqrt{2 / \pi}-$ $\sqrt{\pi / 2} c_{*}^{2} \log \left(\sqrt{\pi e / 32} c_{*}\right)$ (dash-dot line) (see equation $(74)$ ) and for $c_{*} \rightarrow \infty$ given by $c_{*}=d_{*}-2 / \sqrt{3 \pi}$ (dashed line).

For $p_{c}^{*}=-0.5$ the hydrodynamic force becomes negative. Note the significant contribution of the forces in the cavity to the total force acting on the wedge.

\section{Free motion during impact of a body with $p_{c}=0$}

In this section, we consider the free motion of a body which starts to penetrate the liquid vertically with initial velocity $V$. Here we only account for a cavity with cavitation pressure $p_{c}=0$. The motions of the inner and outer contact points are given by equations (35)-(37), (47)-(49) where the vertical motion of the body is governed by Newton's second law. To leading order for small deadrise angle Newton's second law gives

$$
L(t)=-m \ddot{h}(t)
$$

where $m$ is the mass of the body and $L(t)$ is the vertical hydrodynamic force defined by (52). Equation (71) is subject to the initial conditions $h(0)=0$ and $\dot{h}(0)=V$. During the Wagner stage we find the vertical motion by substituting the hydrodynamic pressure (18) in equation (71). Integrating this equation twice in time we obtain

$$
\frac{m}{\varrho}(V t-h)=\frac{\pi}{2} \int_{0}^{h} d^{2}\left(h^{\prime}\right) \mathrm{d} h^{\prime},
$$


where $d=d(h)$ is the position of the outer contact point as a function of the penetration depth given implicitly in equation (17). It can be shown using equation (19) that the cavitation stage starts when the mass $m$ of the body is equal to the added mass induced by the disturbed flow given by $\frac{\pi}{2} \varrho d^{2}$ (see [10]). Furthermore, at this time the speed of the body $\dot{h}$ has reduced to exactly half of the initial speed of the body, $V$.

At the cavitation stage, equations (52) and (71) together with (29) provide

$$
\frac{m}{\pi \varrho}=\frac{C+D}{2}-C \frac{K(q)}{E(q)}, \quad q=\sqrt{1-C / D} .
$$

For $p_{c}=0$ we have the curious situation that the variable to be determined by equation (71), $\ddot{h}$, does not appear in equation (73). The body acceleration $\ddot{h}(t)$ is determined by equation (36). Equation (73) provides the coordinate $c_{*}=c \sqrt{\varrho / m}$ of the separation point as a function of $d_{*}=d \sqrt{\varrho / m}$ independent of the shape of the body. This function is plotted in Figure 11. At the beginning of the cavitation stage we have the following asymptotic behaviour as $c_{*} \rightarrow 0$ :

$$
d_{*}=\sqrt{\frac{2}{\pi}}-\sqrt{\frac{\pi}{2}} c_{*}^{2} \log \left(\sqrt{\frac{\pi e}{32}} c_{*}\right)+O\left(c_{*}^{4} \log ^{2}\left(c_{*}\right)\right) .
$$

The asymptotic formula (74) is consistent with (63) to leading order only, i.e. the coefficients in front of $c_{*}^{2} \log \left(c_{*}\right)$ in these formulas are identical but not the coefficients in front of $c_{*}^{2}$. It follows from (36) that $\ddot{h}\left(t_{0}^{-}\right)=\ddot{h}\left(t_{0}^{+}\right)$and $\dddot{h}\left(t_{0}^{-}\right)=\dddot{h}\left(t_{0}^{+}\right)$but $\left|\dddot{h}\left(t_{0}^{+}\right)\right|=\infty$. For large $c_{*}$ we obtain from (73) that the size of the region in contact with the fluid for $x>0$ is $d_{*}-c_{*}=2 / \sqrt{3 \pi}+O\left(c_{*}^{-2}\right)$ as $c_{*} \rightarrow \infty$. Explicit forms for $A(t)$ and $B(t)$ are given by equations (35), (47) and (73), which are

$$
A=\frac{m}{\pi \varrho}(\dot{h}-V), \quad B=\frac{m}{\pi \varrho}(h-V t) .
$$

The overall structure of the equations is as follows: Equation (36) and the timederivatives of equations (48) and (73) give us a system of ordinary differential equations for $C, D$ and $h$. The functions $U$ and $S$ are obtained by solving the integral equations (37) and (49).

\subsection{Free entry of a parabola}

We investigate the free fall of a parabola onto a liquid free surface and its entry into the liquid. For the formulation of the problem in non-dimensional variables we choose $\sqrt{m / \varrho}$ as the lengthscale and $m /(\varrho V R)$ as the timescale where $R$ is the radius of curvature of the parabola at its lowest point. The penetration depth is much smaller than the horizontal extent of the wetted region provided $\sqrt{m / \varrho} / R \ll 1$. In the Wagner stage, the pressure at the lowest point obtained from (18) reads

$$
p_{0}^{*}=\frac{1-2 \pi h_{*}}{\sqrt{h_{*}}\left(1+2 \pi h_{*}\right)^{3}}, \quad t_{*}=\pi h_{*}^{2}+h_{*} .
$$




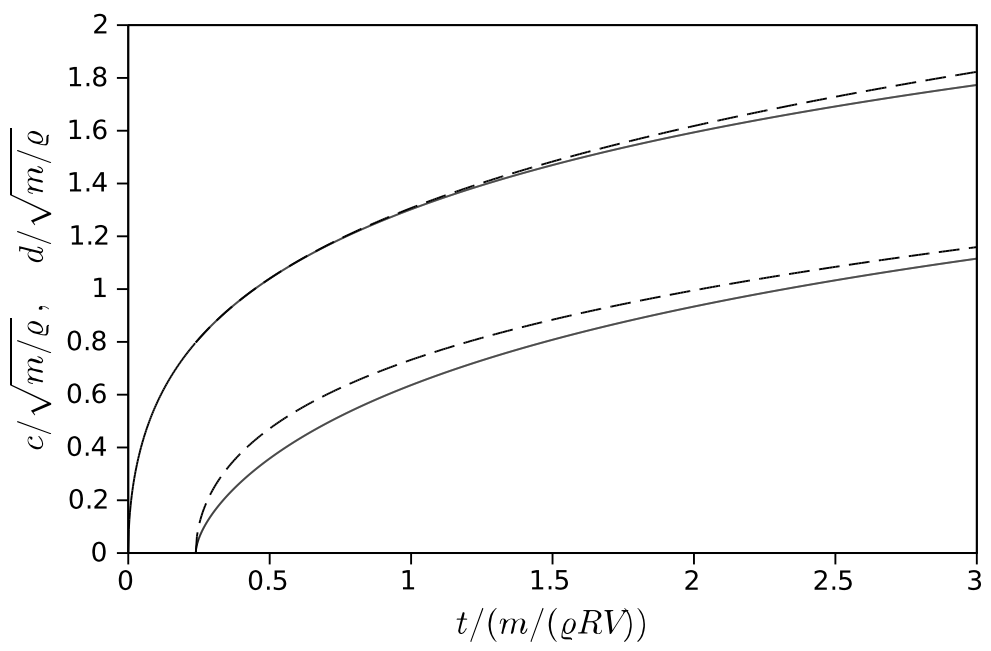

Fig. 12 The coordinates of the contact points, $c(t)$ and $d(t)$, as functions of time (solid lines) for the free entry of a parabola. For the model without a cavity, the positions $x=d(t)$ and $x=\hat{c}(t)$ where $p(\hat{c}, 0, t)=0$ are shown as dashed lines.

Consequently, for $p_{c}=0$ the cavitation stage starts at time $t_{*}=3 /(4 \pi) \approx 0.24$. Note that in this case a cavity develops for any $V, R$ and $m$ if $\sqrt{m / \varrho} / R \ll 1$. In the model without a cavity, the hydrodynamic pressure has a minimum, $p_{0 \min }^{*}=-0.068$, at time $t_{*}=0.508$. Hence, a cavity at vapour pressure $p_{c}=$ $-99 \mathrm{kPa}$ develops only if the parameters $V, R$ and $m$ satisfy $R^{2} V^{4} m^{-1}>$ $2120 \mathrm{~m}^{6} \mathrm{~s}^{-4} \mathrm{~kg}^{-1}$.

Computations in the cavitation stage need to be done only once since the problem in non-dimensional variables does not depend on any parameter. Figure 12 shows the coordinates of the inner and outer contact points $x=c(t)$ and $x=d(t)$. The position $x=c(t)$ is compared with $x=\hat{c}(t)$ at which the hydrodynamic pressure on the body surface is zero, i.e. $p(\hat{c}, 0, t)=0$, in the model without a cavity. Figure 12 shows that $\hat{c}$ increases quicker than $c$ at the beginning of the cavitation stage, but later the difference becomes smaller. The differences in the motions of the Wagner contact points are small throughout the cavitation stage.

Figure 13 shows that the difference in the penetration depths predicted by these two models is rather small at the beginning of the cavitation stage (note that $\left.t_{0} /(m /(\varrho R V)) \approx 0.24\right)$. This is due to the small difference in the hydrodynamic loads by the models during the cavitation stage. Later on the difference in $h(t)$ increases. 


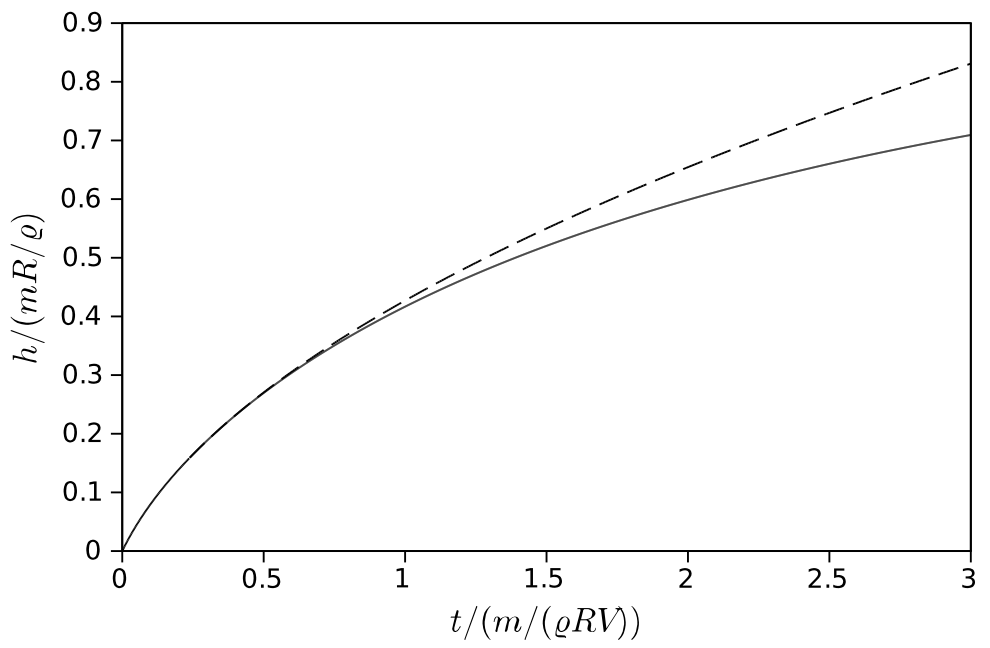

Fig. 13 The penetration depth $h(t)$ as a function of time for the models with cavity (solid line) and without a cavity (dashed line) for $p_{c}=0$ for the free impact of a parabola.

\subsection{Free entry of a wedge}

We investigate the free fall of a wedge onto a liquid free surface. For the formulation of the problem in non-dimensional variables we choose $\sqrt{m / \varrho}$ as the lengthscale and $\varepsilon \sqrt{m / \varrho} / V$ as the timescale, where the deadrise slope $\varepsilon$ of the wedge has to be small. The problem in non-dimensional variables does not depend on any parameter. In the Wagner stage, the pressure at the lowest point obtained from (18) can be written as

$$
p_{0}^{*}=\frac{\pi\left(1-\pi^{3} h_{*}^{2} / 8\right)}{2\left(1+\pi^{3} h_{*}^{2} / 8\right)^{3}}, \quad t_{*}=\frac{\pi^{3}}{24} h_{*}^{3}+h_{*} .
$$

Consequently, for $p_{c}=0$ the cavitation stage starts at time $t_{*} \approx 0.68$. Note that in this case a cavity develops for any $V, \varepsilon$ and $m$. In the model without a cavity, the hydrodynamic pressure has a minimum, $p_{0 \text { min }}^{*}=-0.058$, at time $t_{*}=1.20$. A cavity at vapour pressure develops only if the parameters $V$ and $\varepsilon$ satisfy $V / \sqrt{\varepsilon}>41.3 \mathrm{~m} \mathrm{~s}^{-1}$. This is more than three times greater than the critical value obtained for forced motion with constant deceleration (see after equation (70)). Hence, under free motion, cavitation at vapour pressure is achieveable for only extremely large impact velocities or small deadrise slopes. Note that the occurance of cavitation does not depend on the mass of the wedge.

Figure 14 shows the motions of the inner and outer contact points $x=c(t)$ and $x=d(t)$. The motion of $x=c(t)$ is compared with the position $x=\hat{c}(t)$ where $p(\hat{c}, 0, t)=0$. Figure 14 shows that $\hat{c}$ increases quicker than $c$ at the 


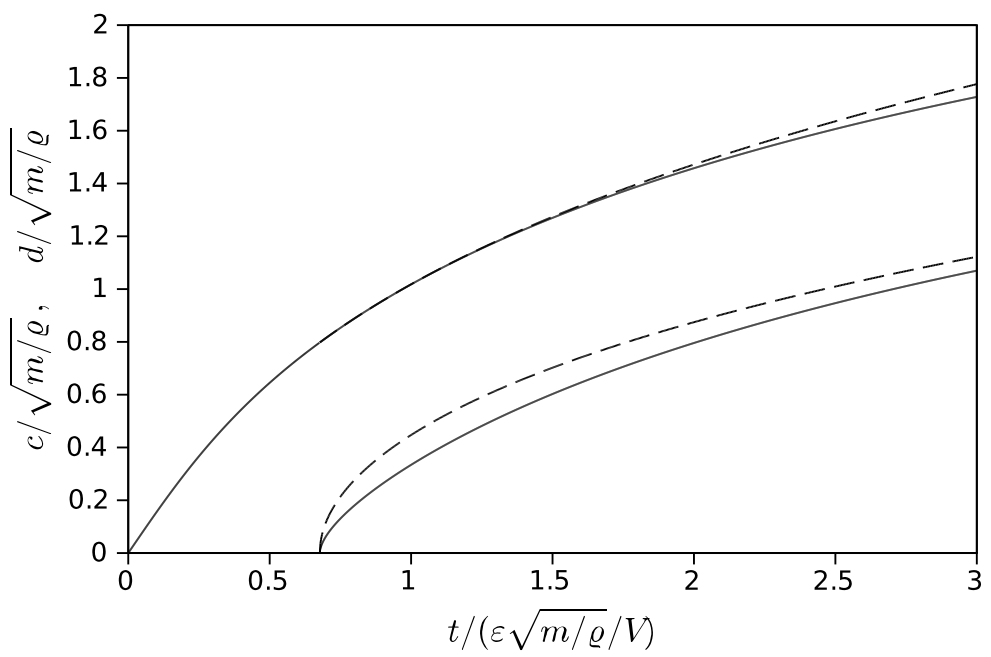

Fig. 14 The coordinates of the contact points $c(t)$ and $d(t)$ as functions of time (solid lines) for the free entry of a wedge. For the model without a cavity, the positions $x=d(t)$ and $x=\hat{c}$ where $p(\hat{c}, 0, t)=0$ are shown as dashed lines.

beginning of the cavitation stage, but later on their difference becomes smaller again. Note that the difference in the motions of the Wagner contact points is rather small. Figure 15 shows that the difference in the penetration depths of the models is small throughout the cavitation stage.

\section{Conclusions and further work}

We considered the vertical impact of a rigid symmetric body onto a liquid free surface, accounting for a cavity region that grows from the centre of the bottom of the body. The model is based on the Wagner theory and the assumption that the hydrodynamic pressure is higher than the pressure in the cavity. The shape of the cavity and the positions of the separation and turnover points are coupled. Six equations for six unknown functions were derived by comparing the acceleration potential, velocity potential and displacement potential at the outer and inner contact points and in the far field. An asymptotic analysis of the initial stage of the cavity formation has been performed. This analysis was helpful to start numerical calculations in the cavitation stage.

Contrasts were drawn between results from the present model and a simpler model in which the cavity formation is suppressed. For forced motion we have shown that in the model without a cavity the expansion of the zone whose pressure is below cavitation pressure is significantly quicker than the expansion of the cavity in the model with cavity. Consequently, the size of the negativepressure zone is not a suitable estimate of the cavity size. 


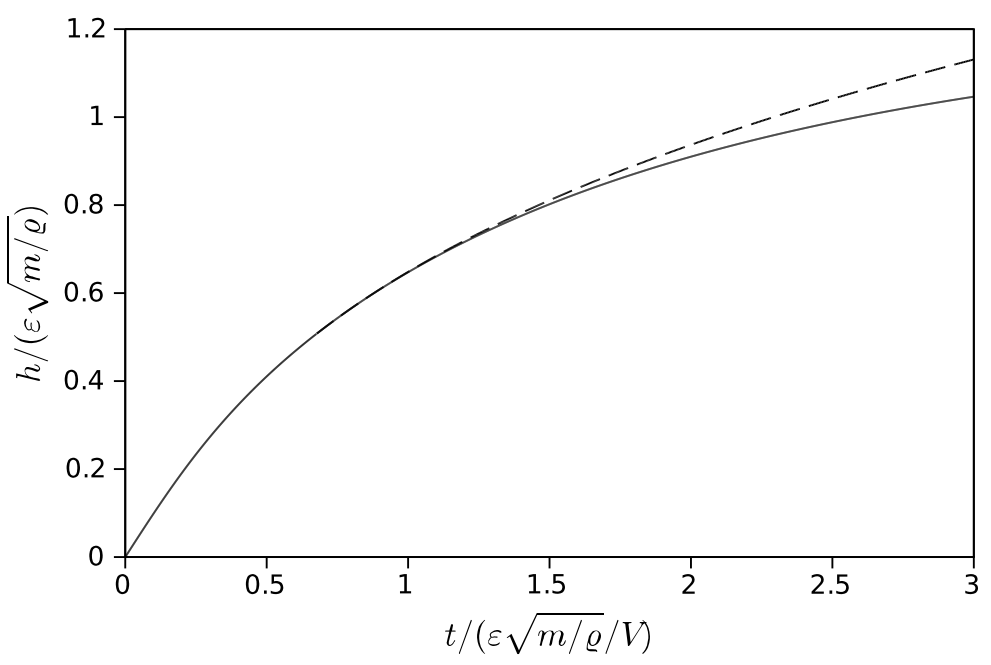

Fig. 15 The penetration depth $h(t)$ as a function of time for the models with cavity at $p_{c}=0$ (solid line) and without a cavity (dashed line) for the free entry of a wedge.

Nevertheless, it is a surprise how quickly the cavity spreads. For forced motion and setting the cavitation pressure to be close to atmospheric, the edge of the cavity has nearly caught up with the contact point within a time of order $V / a$. Although we could not compute up to the time of complete separation of the fluid, the substantial cavity is a zone available for dissolved gases or ambient bubbles to congregate as, for example, a foam layer, but this is not considered here.

If the body is free to move under the hydrodynamic force, we found that results change little between the model that includes cavitation and the model without a cavity. Whether or not a cavity occurs strongly depends on the initial impact velocity, for which we have found helpful bounds. Extremely large impact velocities, or small deadrise slopes, satisfy these bounds.

We finally discuss how the present model can be extended. Results by this model are shown only for constant cavitation pressure. However, the same equations hold if the cavitation pressure varies as a prescribed function of time. Elasticity of the body may promote cavitation (see [19]) which can be analysed with an extended model of the one shown in this paper. Furthermore, the present model can be extended to problems of oblique body impact (see [14] and [15]). The occurance of cavity formation for oblique impact is more likely than for vertical impact. However, in such a non-symmetric problem four contact points have to be treated separately which makes the analysis much more difficult. The investigation of the influence of viscosity is another matter for further investigation. 
Acknowledgements M.R. was financially supported by the International Research Training Group 1529 "Mathematical Fluid Dynamics" at TU Darmstadt. A.K. acknowledges the support by the NICOP research grant "Fundamental analysis of the water exit problem" (N62909-13-1-N274). The authors thank Alan Tassin for his helpful discussions. The authors are grateful to the referees for their careful readings and comments, which helped to improve an earlier version of this paper.

\section{A A Solution of the MBVP}

In this Appendix we present the solution of the problem (20)-(26) such that inequalities (15) and (16) are satisfied. The problem (20) - (24) can be reformulated as a Dirichlet problem for the analytic function

$$
\omega_{0}^{*}(z)=\left(\varphi_{x}-i \varphi_{y}-i \ddot{h}\right) g(z), \quad z=x+i y \quad(y<0),
$$

where $g(z)$ is the characteristic function defined in (27). The equations (21)-(26) imply that $\omega_{0}^{*}(z)$ is bounded at $z= \pm c$, has simple poles at $z= \pm d$ and its real part is zero on the $x$-axis. Such a function has the form

$$
\omega_{0}^{*}(z)=i \frac{Q(z)}{z^{2}-d^{2}},
$$

where $Q(z)=\sum_{k=0}^{4} \alpha_{k} z^{k}$ is a polyomial with real coefficients. This polynomial is of order four since $\omega_{0}^{*}(z) \stackrel{k}{=} O\left(z^{2}\right)$ as $z \rightarrow \infty$. As the flow is symmetric it can be shown that $\omega_{0}^{*}(z)$ satisfies the symmetry condition $\overline{\omega_{0}^{*}(z)}=-\omega_{0}^{*}(-\bar{z})$ where the bar denotes the complex conjugate of a complex number. It follows from this symmetry condition that $\alpha_{1}=\alpha_{3}=0$. We find the following formula for the complex acceleration $\omega_{0}(z)=\varphi_{x}-i \varphi_{y}$ by using the definition of $\omega_{0}^{*}(z)$ and equation $(79)$ :

$$
\omega_{0}(z)=i \ddot{h}\left(1+\frac{\beta_{0}+\beta_{1}\left(z^{2}-c^{2}\right)+\beta_{2}\left(z^{2}-c^{2}\right)^{2}}{\left(z^{2}-d^{2}\right)^{3 / 2} \sqrt{z^{2}-c^{2}}}\right)
$$

with real coefficients $\beta_{i}$. Since $\omega_{0}(z)$ decays in the far field we have $\beta_{2}=-1$.

We investigate the pressure behaviour at the detachment point $z=c$. The pressure gradient along the contact region $c<x<d, y=0$, is given by the real part of $\omega_{0}(z)$ in (80) together with the linearised Bernoulli's equation (4). The asymptotic behaviour of $p_{x}(x, 0, t), c<x<d$, at $x=c, y=0$, is

$$
p_{x}(x, 0, t) \sim-\frac{\beta_{0} \ddot{h}}{\sqrt{2} c\left(d^{2}-c^{2}\right)^{3 / 2}}(x-c)^{-1 / 2} \quad(x \rightarrow c) .
$$

If $\beta_{0} \ddot{h}>0$, the pressure is below the cavitation pressure $p_{c}$ in the vicinity of the detachment point $c$, which is a contradiction to the inequality (16). Therefore,

$$
\beta_{0} \ddot{h} \leq 0
$$

Now we analyse the free surface elevation $y=\eta(x, t)$ in the cavity region $-c<x<c$ close to the separation point $x=c$. Kutta's condition guarantees that the fluid leaves the body tangentially (see [19]). The imaginary part of (80) together with the kinematic boundary condition (12) gives us the asymptotic behaviour of the vertical fluid acceleration on the free surface at $x=c$ :

$$
\eta_{t t}(x, 0, t) \sim-\frac{\beta_{0} \ddot{h}}{\sqrt{2} c\left(d^{2}-c^{2}\right)^{3 / 2}}(c-x)^{-1 / 2} \quad(x \rightarrow c) .
$$

The value $\beta_{0} \ddot{h}$ in (83) is responsible, for whether or not the fluid intersects the body at the separation point. For a smooth body, it follows from (15), (82) and (83) that both conditions 
on the behaviours of the hydrodynamic pressure and the cavity thickness are satisfied only with $\beta_{0}=0$.

The value of $\beta_{1}$ determines the value of the acceleration potential $\varphi(x, y, t)$ in the cavity region $|x|<c, y=0$. Equation (23) and the far-field condition (24) imply that $\int_{-\infty}^{0} \varphi_{y}(0, y, t) \mathrm{d} y=-p_{c} / \varrho$, where $\varphi_{y}$ is given by the imaginary part of $\omega_{0}(z)$ in $(80)$. Hence,

$$
\beta_{1}=2 d^{2}-c^{2}-\frac{1}{E(q)}\left(\frac{p_{c}}{\varrho \ddot{h}} d+c^{2} K(q)\right), \quad q=\sqrt{1-\frac{c^{2}}{d^{2}}},
$$

where $K(q)$ and $E(q)$ are the complete elliptic integrals of the first and second kind, respectively.

\section{References}

1. Crighton, D.G.: The Kutta condition in unsteady flow. Ann. Rev. Fluid Mech. 17, 411-445 (1985)

2. Faltinsen, O.: The effect of hydroelasticity on ship slamming. Phil. Trans. R. Soc. Lond. A 355(1724), 575-591 (1997)

3. Gakhov, F.D.: Boundary Value Problems. Pergamon, Oxford (1966)

4. Hicks, P.D., Ermanyuk, E.V., Gavrilov, N.V., Purvis, R.: Air trapping at impact of a rigid sphere onto a liquid. J. Fluid. Mech. 695, 310-320 (2012)

5. Howison, S., Morgan, J., Ockendon, J.: Patch cavitation in flow past a rigid body. In: Bubble Dynamics and Interface Phenomena, pp. 219-226. Springer (1994)

6. Howison, S.D., Ockendon, J.R., Wilson, S.K.: Incompressible water-entry problems at small deadrise angles. J. Fluid Mech. 222, 215-230 (1991)

7. Kaye, G., Laby, T.: Tables of physical and chemical constants. Longman, Essex (1995)

8. Korobkin, A.A.: Low-pressure zones under a liquid-solid impact. In: Bubble Dynamics and Interface Phenomena, pp. 375-381. Springer (1994)

9. Korobkin, A.A.: Water impact problems in ship hydrodynamics. In: M. Ohkusu (ed.) Advances in Marine Hydrodynamics, pp. 323-371. Computational Mechanics, Boston (1996)

10. Korobkin, A.A.: Cavitation in liquid impact problems. In: Fifth International Symposium on Cavitation, pp. 1-4. Osaka (2003)

11. Korobkin, A.A.: A linearized model of water exit. J. Fluid Mech. 737, 368-386 (2013)

12. Li, L., Papadopolous, D.P., Smith, F.T., Wu, G.X.: Rapid plunging of a body partly submerged in water. J. Eng. Math. 42, 303-319 (2002)

13. Logvinovich, G.V., Yakimov, Y.L.: Submergence of bodies in liquid with large velocities. In: L.I. Sedov, G.Y. Stepanov (eds.) Neustanovivshiesia Techenia Vody s Bol'shimi Skorostyami Trudy Mezhdunarodnogo Simposiuma v Leningrade, pp. 85-92. Nauka, Moscow (1973)

14. Moore, M.R., Howison, S.D., Ockendon, J.R., Oliver, J.M.: Three-dimensional oblique water-entry problems at small deadrise angles. J. Fluid Mech. 711, 259-280 (2012)

15. Moore, M.R., Howison, S.D., Ockendon, J.R., Oliver, J.M.: A note on oblique water entry. J. Eng. Math. 81(1), 67-74 (2013)

16. Oliver, J.M.: Water entry and related problems. D.Phil thesis, University of Oxford (2002)

17. Reinhard, M., Korobkin, A., Cooker, M.J.: The bounce of a blunt body from a water surface at high horizontal speed. In: 27th International Workshop on Water Waves and Floating Bodies, pp. 153-156. Technical University of Denmark, Copenhagen (2012)

18. Reinhard, M., Korobkin, A.A., Cooker, M.J.: Elastic plate impact into water at high horizontal speed with early water detachment. In: 6th International Conference on Hydroelasticity in Marine Technology 2012, pp. 1-10. University of Tokyo Press, Tokyo (2012)

19. Reinhard, M.V.: Free elastic plate impact into water. PhD thesis, University of East Anglia (2013) 
20. Smith, F.T., Li, L., Wu, G.X.: Air cushioning with a lubrication/inviscid balance. J. Fluid Mech. 482, 291-318 (2003)

21. Wagner, H.: Über Stoß- und Gleitvorgänge an der Oberfläche von Flüssigkeiten. ZAMM 12, 193-215 (1932)

22. Wilson, S.K.: A mathematical model for the initial stages of fluid impact in the presence of a cushioning fluid layer. J. Eng. Math. 25, 265-285 (1991) 